ARTICLE

\title{
Transformation of tenofovir into stable ProTide nanocrystals with long-acting pharmacokinetic profiles
}

Denise A. Cobb ${ }^{1}$, Nathan Smith ${ }^{1}$, Suyash Deodhar (10 ${ }^{1}$, Aditya N. Bade ${ }^{1}$, Nagsen Gautam², Bhagya Laxmi Dyavar Shetty' ${ }^{1}$ JoEllyn McMillan', Yazen Alnouti², Samuel M. Cohen (10 ${ }^{3}$, Howard E. Gendelman (iD) ${ }^{1,2} \&$ Benson Edagwa (i) ${ }^{1 凶}$

Treatment and prevention of human immunodeficiency virus type one (HIV-1) infection was transformed through widespread use of antiretroviral therapy (ART). However, ART has limitations in requiring life-long daily adherence. Such limitations have led to the creation of long-acting (LA) ART. While nucleoside reverse transcriptase inhibitors (NRTI) remain the ART backbone, to the best of our knowledge, none have been converted into LA agents. To these ends, we transformed tenofovir (TFV) into LA surfactant stabilized aqueous prodrug nanocrystals (referred to as NM1TFV and NM2TFV), enhancing intracellular drug uptake and retention. A single intramuscular injection of NM1TFV, NM2TFV, or a nanoformulated tenofovir alafenamide (NTAF) at $75 \mathrm{mg} / \mathrm{kg}$ TFV equivalents to Sprague Dawley rats sustains active TFV-diphosphate (TFV-DP) levels $\geq$ four times the $90 \%$ effective dose for two months. NM1TFV, NM2TFV and NTAF elicit TFV-DP levels of 11,276, 1,651, and $397 \mathrm{fmol} / \mathrm{g}$ in rectal tissue, respectively. These results are a significant step towards a LA TFV ProTide.

\footnotetext{
${ }^{1}$ Department of Pharmacology and Experimental Neuroscience, University of Nebraska Medical Center, Omaha, NE, USA. ${ }^{2}$ Department of Pharmaceutical Sciences, University of Nebraska Medical Center, Omaha, NE, USA. ${ }^{3}$ Department of Pathology and Microbiology, University of Nebraska Medical Center,

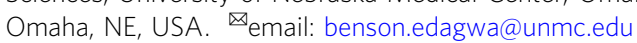


D espite advances made by the broad use of antiretroviral therapy (ART) for the treatment and prevention of human immunodeficiency virus type one (HIV-1) infection adherence to daily regimens remains a major obstacle in achieving sustained viral suppression and in preventing viral transmission ${ }^{1}$. It is generally perceived that extended-release long-acting (LA) ART designed to ease patient dosing and synchronize CD4+ T cell and HIV-1 load measurements could positively impact treatment outcomes. Notably, LA formulations are widely used as contraceptives and antipsychotics and have already shown improvement in regimen adherence ${ }^{2,3}$. This idea has recently been forged for HIV-1 therapy as rilpivirine and cabotegravir (RPV and CAB) LA are now approved for human use $^{4,5}$ in Canada, Europe, and the US $^{6}$ as injectable solid drug nanosuspensions. The injectables are administered monthly or every other month ${ }^{7,8}$. Such LA antiretroviral drug (ARV) delivery strategies could serve to benefit the most vulnerable populations such as intravenous drug users, children, and pregnant women. However, limitations of CAB and RPV LA include the requirement for large injection volumes, short dosing intervals, none of them are active against major HIV co-infections such as hepatitis $\mathrm{B}$ and are also characterized by limited drug access to cellular and tissue infectious reservoirs ${ }^{9-11}$. Attempts to overcome these have led to the transformation of native ARVs into nanoformulated prodrugs designated as slow effective release antiretroviral therapy (LASER ART). These are homogeneous dispersions of solid prodrug nanocrystals stabilized by aqueous surfactant solutions ${ }^{12-15}$. The LASER ART prodrug nanocrystals provide slow drug dissolution and rapid transport across biological membranes. This can achieve sustained plasma and tissue drug concentrations above the $90 \%$ effective concentration (EC90) for periods of up to one year ${ }^{16}$.

The target product profile of compounds suitable for parenteral LA formulations includes high potency, lower injection volumes, extended dosage intervals, low aqueous solubility, drug stability, slow dissolution and release, and extended elimination times. All can be achieved, in part, through the creation of prodrugs. For example, the ProTide prodrug technology has enabled safe and efficient intracellular delivery of phosphorylated nucleoside and nucleotide analogs in virus target cells ${ }^{17}$. The application of ProTide technology to tenofovir (TFV) resulted in the discovery of TFV alafenamide (TAF), a phosphoramidate prodrug. Compared to tenofovir disoproxil fumarate (TDF), TAF is more potent and its safety profile makes it a better candidate for transformation into an LA formulation ${ }^{18,19}$. However, TAF is susceptible to hydrolytic degradation, by non-enzymatic hydrolysis in an aqueous environment ${ }^{20,21}$. Attempts to modify formulation excipients or $\mathrm{pH}$ to affect TAF stability are yet to demonstrate improved pharmacokinetic (PK) outcomes ${ }^{22-25}$. We now describe an improved ProTide strategy that masks the phosphate groups in TFV with cleavable hydrophobic amino acid ester lipids with synergistic effects on nucleoside analogs ${ }^{26,27}$. These modifications affect drug efficacy, prodrug aqueous stability, dissolution, half-life, and safety ${ }^{26-28}$.

In this work, we describe the preclinical development of two lipophilic aqueous stable TFV ProTide nanoformulations referred to as NM1TFV and NM2TFV. The formulations exhibit improved intracellular drug delivery, retention, and protection against HIV-1 infection in vitro when compared against a nanoformulated TAF control (NTAF). Notably, a single intramuscular injection of NM1TFV or NM2TFV to Sprague Dawley (SD) rats at $75 \mathrm{mg} / \mathrm{kg}$ TFV equivalents sustains TFV-DP levels above the EC90 in peripheral blood mononuclear cells (PBMC) for two months with no recorded adverse events. The lead NM1TFV sustains tissue TFV-DP levels four times higher than the known drug $\mathrm{EC}_{90}$ of $16-48 \mathrm{fmol} / 10^{6}$ cells linked to HIV-1 prevention $^{29}$. Our studies provide proof of concept that conversion of TFV into stable ProTide formulations can provide extended drug dosing intervals.

\section{Results}

Synthesis and characterization of TFV ProTides. We created lipophilic ProTides of TFV bearing phenylalanine and alanine amino acid esters (coined as M1TFV and M2TFV) by replacing optimal short chain alkyl ester groups utilized by conventional ProTide strategies $28,30,31$. Bulky residues were used to create the ProTides (Fig. 1). The docosanol masking ester motif was selected based on inherent lipophilicity and synergy with nucleoside analogs ${ }^{26,27}$. M1TFV and M2TFV were synthesized by coupling a phenylalanyl or alanyl docosyl ester (Fig. 1a, b) to monophenyl TFV in the presence of $\mathrm{Et}_{3} \mathrm{~N}$ to yield the desired compounds with chemical yields of $50-65 \%$ and purities of $98.4 \%$ for M1TFV, and $94.9 \%$ for M2TFV (Supplementary Figs. 1-3). Since the conjugation step is moisture sensitive, further improvements to the chemical yields could be achieved by either optimizing the coupling reagents or reaction vessels. Infusion of the synthesized compounds into an Autoflex maX MALDI-TOF/TOF mass spectrometer confirmed molecular mass ions of 477.022 for TAF, 819.42 for M1TFV, and 743.361 for M2TFV (Supplementary Figs. 4-6). Conversion of TFV into ProTides altered the physicochemical properties of TFV. TAF, M1TFV and M2TFV exhibited aqueous solubilities of 5.52, 0.002 , and $0.007 \mathrm{mg} / \mathrm{mL}$, respectively. In contrast, the solubility of the prodrugs in 1-octanol were $4.85,29.2$, and $20.9 \mathrm{mg} / \mathrm{mL}$ for TAF, M1TFV, and M2TFV, respectively. This confirmed increases in prodrug lipophilicity (Fig. 1c). To determine the effect of prodrug modifications and nanoparticle dissociation on compound chemical stability, $1 \mu \mathrm{M}$ NM1TFV or NTAF formulations were incubated for $24 \mathrm{~h}$ in buffers of increasing $\mathrm{pH}$. These included $\mathrm{pH}$ of 2.0 ( $0.1 \%$ formic acid), $6.0(7.5 \mathrm{mM}$ ammonium acetate), 7.0 (PBS), 8 (7.5 mM ammonium bicarbonate), and 10.3 ( $0.1 \%$ ammonium hydroxide). No statistical differences in prodrug stability upon nanoparticle dissociation and prodrug dissolution were observed between NM1TFV and $\mathrm{NTAF}$ at $\mathrm{pH} 2.0$ from the 0 to $2 \mathrm{~h}$ timepoints, at $\mathrm{pH} 6.0$ from the 0 to $12 \mathrm{~h}$ timepoints, or at $\mathrm{pH} 7.0$ at any timepoint (Supplementary Fig. 7 and Supplementary Table 1). However, dissociated NM1TFV demonstrated significantly greater prodrug stability at $\mathrm{pH} 2.0$ at the 6 and $12 \mathrm{~h}$ timepoints, $\mathrm{pH} 6.0$ at the $24 \mathrm{~h}$ timepoint, and at $\mathrm{pH} 8.0$ and 10.3 at all timepoints (Supplementary Fig. 7 and Supplementary Table 1), suggesting that unlike TAF that is hydrolytically unstable at basic $\mathrm{pH}, \mathrm{M} 1 \mathrm{TFV}$ confers enhanced prodrug stability over a broader $\mathrm{pH}$ range. It is also worth noting that stability tests were performed on dissociated formulation particles upon dilution and that M1TFV is stable within NM1TFV solid drug nanoparticles. The two ProTides were further characterized by ${ }^{1} \mathrm{H}$ and ${ }^{31} \mathrm{P}$ NMR. The triplet at $0.87 \mathrm{ppm}$ and multiplets at $1.21-1.35 \mathrm{ppm}$ on the spectrum of M1TFV correspond to the terminal methyl and methylene protons of the docosyl ester, respectively. Chemical shifts at 7.04-7.38 ppm represent the aryl and phenylalanine masking promoieties (Fig. 1e). For M2TFV, the triplet at $0.87 \mathrm{ppm}$ and multiplets at $1.20-1.38 \mathrm{ppm}$ correspond to the terminal methyl and methylene protons of the docosyl ester, respectively. The triplets at 7.10, 7.21, 7.31, and doublets at 6.99 and $7.14 \mathrm{ppm}$ represent the aryl and phenol groups (Fig. 1e). The two peaks in the phosphorous NMR spectra indicated a 1:1 mixture of the $\mathrm{R}$ and $S$ diastereomers at the phosphorous chiral center ${ }^{32}$ (Supplementary Fig. 8). Even though phosphorous chirality of ProTides has been shown to affect potency, our studies used the mixture of isomers since the chirality would be lost following 
a

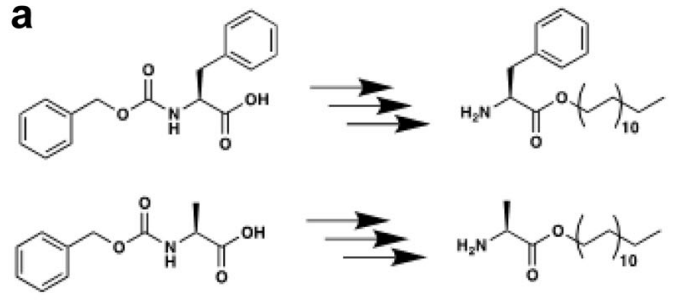

C

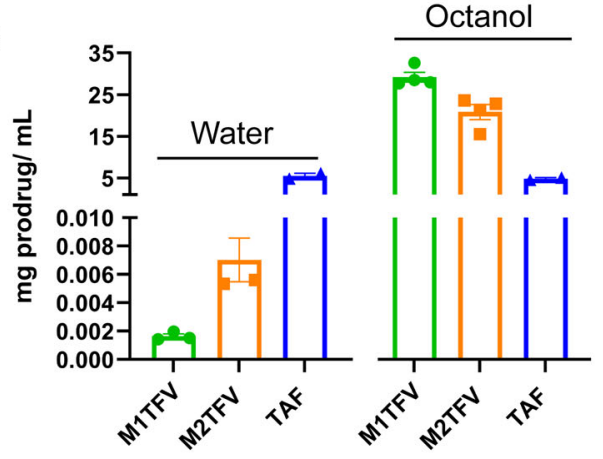

b

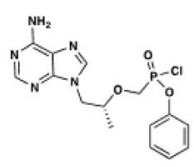
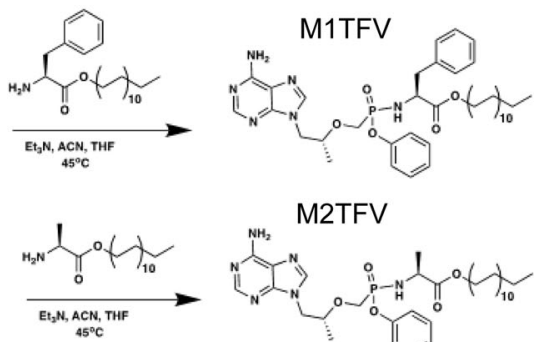

M2TFV

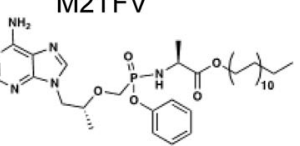

d

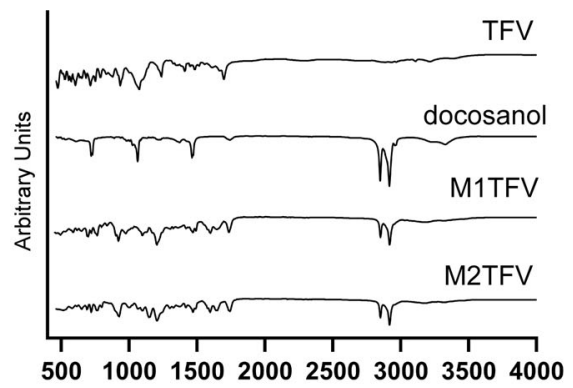

$\mathrm{cm}^{-1}$

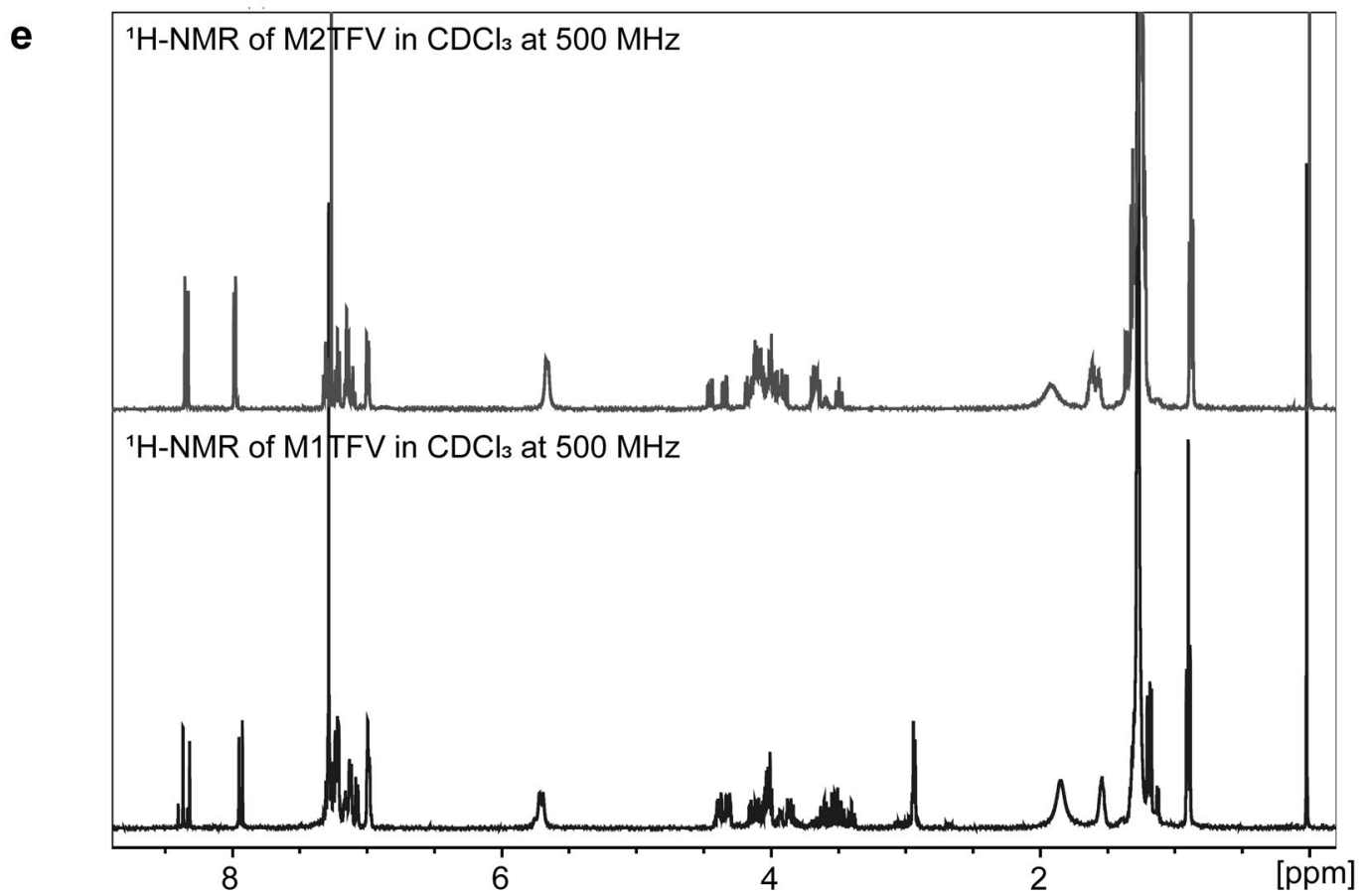

Fig. 1 Characterization of TFV ProTides. a Synthesis of phenyl alanine and alanine derivatizing promoieties. $\mathbf{b}$ Conjugation of the amino acid esters to activated monophenyl TFV to form M1TFV and M2TFV prodrugs. c Aqueous and octanol solubility of M1TFV and M2TFV demonstrate the decreased aqueous solubility of M1TFV (green) and M2TFV (orange), and increased octanol solubility compared to TAF (blue). Values reported are the mean \pm SEM of three replicates. d FT-IR Absorption bands at 2,844 and 2,912 $\mathrm{cm}^{-1}$ illustrate the asymmetric and symmetric $\mathrm{C}-\mathrm{H}$ stretches from the long chain fatty acid. e An overlay of the proton NMR spectra confirmed the synthesis of M1TFV (below) and M2TFV (above). Specifically, the triplet peak at 0.87 ppm and multiplets at 1.21-1.35 ppm correspond to the terminal methyl and methylene protons of the docosyl ester on M1TFV, respectively. Chemical shifts at 7.04-7.38 ppm represent the aryl and phenylalanine masking promoieties. For M2TFV, the triplet at 0.87 ppm and multiplets at 1.20-1.38 ppm correspond to the terminal methyl and methylene protons of the docosyl ester, respectively. Chemical shifts at 6.99, 7.10, 7.14, 7.21, and 7.31 ppm represent the aryl masking promoiety. a-e Experiments were repeated independently five times with equivalent results.

intracellular cleavage of the masking groups. Further chemical characterization of the ProTides by FTIR showed absorption bands at 2,844 and 2,912 $\mathrm{cm}^{-1}$ corresponding to asymmetric and symmetric $\mathrm{C}-\mathrm{H}$ stretches from the long-chain fatty alcohol (Fig. 1d).
Characterization of nanoformulated TFV ProTides. Nanocrystal formulation strategy has so far resulted in the successful development of several monthly IM injectable products that include CAB and RPV LA. Nanocrystals provide enhanced drug dissolution, high loading while obviating organic solvent usage 

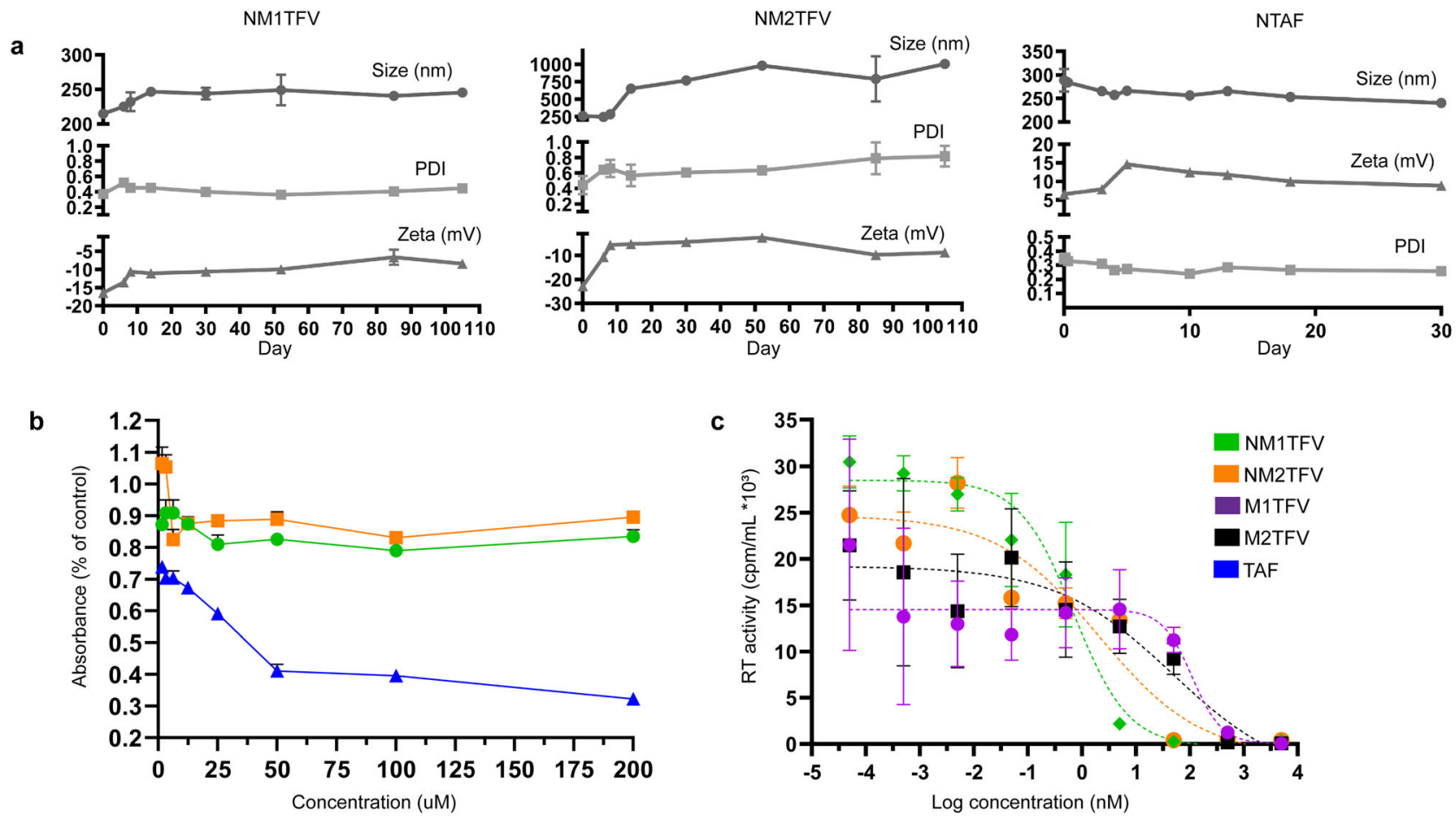

Fig. 2 Nanoformulation of TFV ProTides. Nanoformulations were synthesized by high-pressure homogenization using poloxamer 407 (P407) as the excipient for NM1TFV/NM2TFV, or P407 and PEG 3350 as excipients for NTAF. a Formulation stability at $25^{\circ} \mathrm{C}$ (up to 110 days) was measured by particle hydrodynamic diameter (size), polydispersity index (PDI), and zeta potential as determined by dynamic light scattering (DLS). Data were independently reproduced three times. $\mathbf{b}$ Cell viability was assessed in MDM by MTT assay $8 \mathrm{~h}$ after NM1TFV, NM2TFV, or TAF treatment over a range of concentrations $(1-200 \mu M)$. Results were normalized to untreated control cells. All results are shown as the mean \pm SEM of four replicates. c IC 50 of M1TFV, M2TFV, and their nanoformulations in MDM was investigated at a range of concentrations $(0.00005-5,000 \mathrm{nM})$ and determined by HIV-1 reverse transcriptase (RT) activity after viral challenge with $\mathrm{HIV}-1_{\mathrm{ADA}}$ at an $\mathrm{MOI}$ of 0.1 . Data were normalized and expressed as a percentage of HIV -1 infected vehicle control \pm SEM; $n=4$ biological replicates. Experiments $\mathbf{b}$, $\mathbf{c}$ were repeated independently two times with equivalent results.

and associated potential toxicities ${ }^{21}$. Aqueous surfactant stabilized M1TFV, M2TFV and TAF nanocrystals (NM1TFV, NM2TFV, and NTAF) were therefore produced by high-pressure homogenization. Drug encapsulation efficiencies for NM1TFV, NM2TFV, and NTAF nanocrystals were $94.6,92.2$, and $78.8 \%$, respectively. The size, polydispersity index (PDI), and zeta potential for NM1TFV, were $215 \pm 10.3 \mathrm{~nm}, 0.30 \pm 0.03$, and $-16.4 \pm 0.6 \mathrm{mV}$, respectively, and remained stable at $25^{\circ} \mathrm{C}$ for at least 90 days (Fig. 2a). The size, polydispersity index (PDI), and zeta potential for NM2TFV, were $261.6 \pm 12.9 \mathrm{~nm}, 0.38 \pm 0.08$, and $-22.7 \pm 0.7 \mathrm{mV}$, respectively, and remained stable at $25^{\circ} \mathrm{C}$ for at least 10 days (Fig. 2a). The size, PDI, and zeta potential for NTAF were $284.3 \pm 14 \mathrm{~nm}, 0.3 \pm 0.005$, and $6.55 \pm 0.15 \mathrm{mV}$, respectively, and remained stable at $25^{\circ} \mathrm{C}$ for at least 30 days (Fig. 2a). We then determined the effect of the prodrugs on mitochondrial function by MTT in MDM. No adverse effects on mitochondrial activity or cell viability were observed at $200 \mu \mathrm{M}$ in MDM for NM1TFV and NM2TFV after incubation with drug for $8 \mathrm{~h}$ (Fig. 2b). In contrast, a decrease in cell viability was observed for TAF at concentrations above $12.5 \mu \mathrm{M}$ (Fig. $2 \mathrm{~b}$ ). We next evaluated the ProTides for antiretroviral activity. The calculated $\mathrm{EC}_{50}$ values for unformulated M1TFV and M2TFV in human monocyte-derived macrophages (MDMs) were $109 \mathrm{nM}$ and $45.75 \mathrm{nM}$, respectively (Fig. 2c). The observed potency is likely linked to poor prodrug solubility upon dilution and precipitation in aqueous culture media. Notably, nanoformulation of the prodrugs significantly improved drug potency, with calculated $\mathrm{EC}_{50}$ values of 0.71 and $2.08 \mathrm{nM}$ for NM1TFV and NM2TFV, respectively (Fig. 2c). The antiretroviral activity of TAF $\left(\mathrm{EC}_{50}\right.$ $0.22 \mathrm{nM})$ following nanoformulation into $\mathrm{NTAF}\left(\mathrm{EC}_{50} 0.44 \mathrm{nM}\right.$,
Supplementary Fig. 9a) was equivalent. In humans, CD4+ T-cell line (CEM-ss CD4+ T-cells), the $\mathrm{EC}_{50}$ of NM1TFV, NM2TFV, and TAF were $28.8,30.0$, and $0.33 \mathrm{nM}$ respectively (Supplementary Fig. 9a, c).

Cellular drug uptake and retention. Delivery of solid drug nanoparticles to macrophages has been shown to improve the half-life of ART through the formation of intracellular and tissue drug storage sites $^{11,12}$. Macrophage nanoparticle uptake and storage are facilitated by their phagocytic and proinflammatory functions, large cytoplasmic to the nuclear ratio for drug storage, and their significant role in viral infection makes them ideal targets for ART ${ }^{16}$. We, therefore, evaluated NM1TFV, NM2TFV, and TAF in MDMs at concentrations of 10 and $100 \mu \mathrm{M}$. It is worth noting that evaluation of TAF in MDMs was performed at $10 \mu \mathrm{M}$ since $100 \mu \mathrm{M}$ was found to be highly toxic to cells. Intracellular prodrug concentrations were evaluated over $8 \mathrm{~h}$. Previous work from our laboratory has shown that maximal drug nanoparticle uptake by MDM occurs within $8 \mathrm{~h}^{12,33,34}$. As shown in Fig. 3a, b, the highest drug uptake was observed with NM1TFV. Intracellular prodrug concentration for NM1TFV at $8 \mathrm{~h}$ after treatment with $10 \mu \mathrm{M}$ of drug was $8.62 \mu \mathrm{g} / 10^{6}$ cells (Fig. 3a), a 4.2- and 22.7-fold higher concentration than that observed for NM2TFV $\left(2.04 \mu \mathrm{g} / 10^{6}\right.$ cells $)$ or TAF $\left(0.38 \mu \mathrm{g} / 10^{6}\right.$ cells), respectively. For $100 \mu \mathrm{M}$ drug treatment after $8 \mathrm{~h}$ (Fig. 3b), intracellular prodrug concentration for NM1TFV was $42.36 \mu \mathrm{g} /$ $10^{6}$ cells, a 2.2 -fold higher concentration than that observed for $\operatorname{NM} 2$ TFV $\left(19.4 \mu \mathrm{g} / 10^{6}\right.$ cells). Visualization of MDMs treated with equimolar drug nanoparticles by TEM revealed greater 


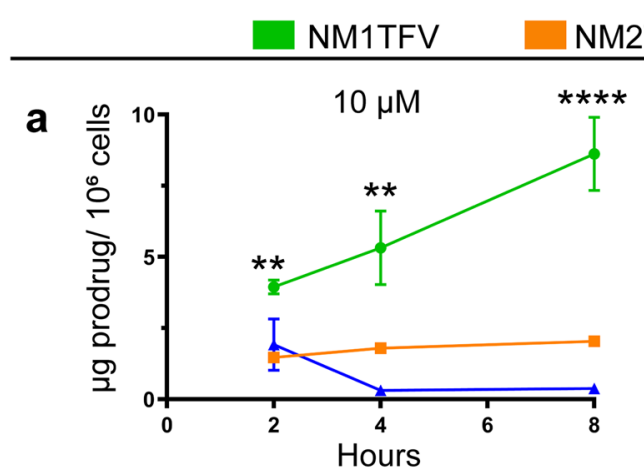

NM2TFV $\square$ TAF
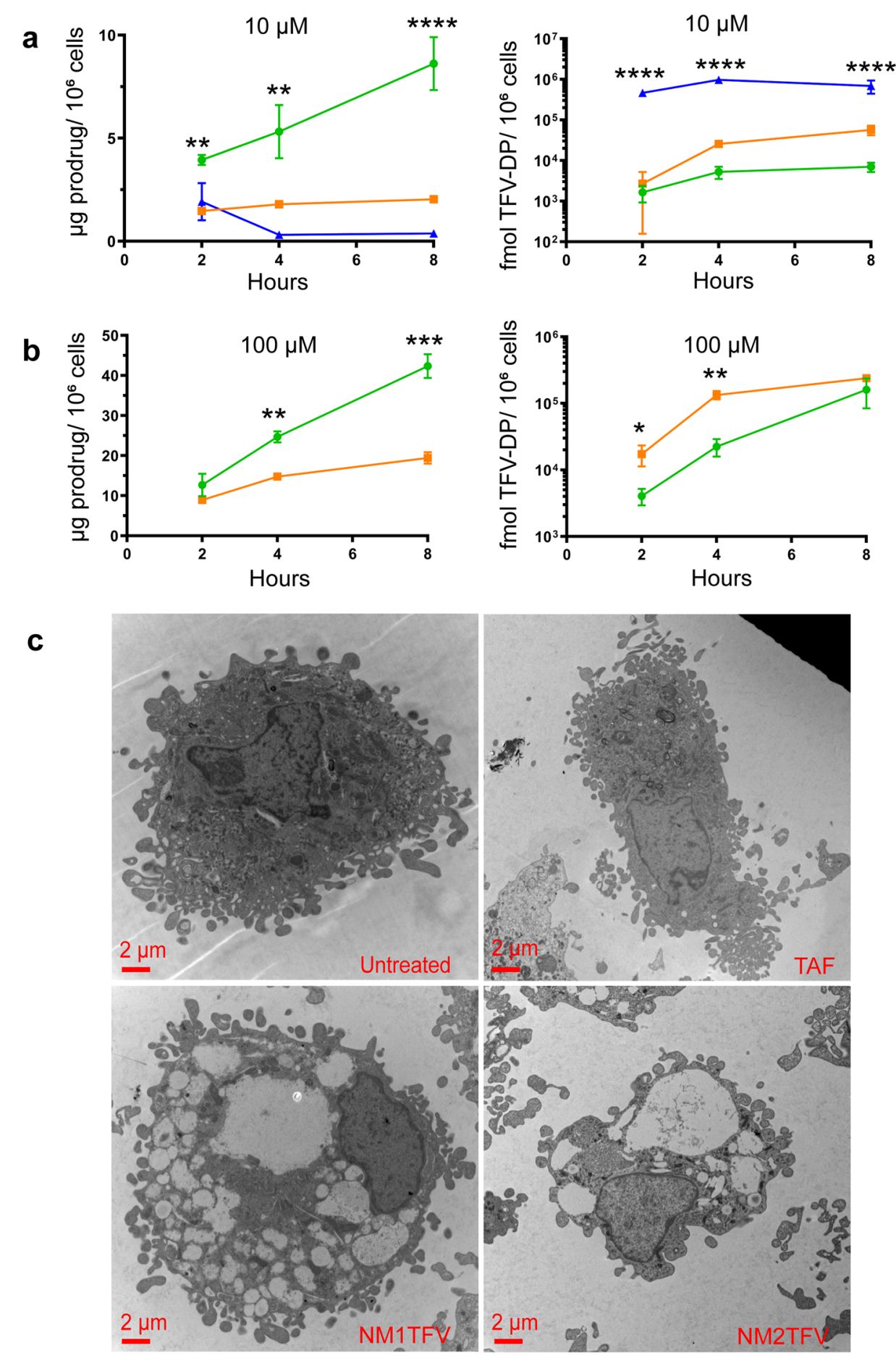

Fig. 3 Cellular uptake. Following a $10 \mu \mathrm{M}\left({ }^{\star \star} P=0.0035,{ }^{\star \star} P=0.0029,{ }^{\star \star \star \star} P<0.0001 \mathrm{NM} 1 \mathrm{TFV}\right.$ vs. NM2TFV prodrug; ${ }^{\star \star \star \star} P<0.0001 \mathrm{NM} 1 \mathrm{TFV}$ vs. TAF TFV$\mathrm{DP})$ or $\mathbf{b} 100 \mu \mathrm{M}$ treatments $\left({ }^{\star \star} P=0.0021,{ }^{\star \star \star} P=0.0003 \mathrm{NM} 1 \mathrm{TFV}\right.$ vs. NM2TFV prodrug; ${ }^{\star} P=0.0273,{ }^{\star \star} P=0.0022 \mathrm{NM} 1 \mathrm{TFV}$ vs. NM2TFV TFV-DP), intracellular prodrug (left) and TFV-DP (right) concentrations in MDM was measured over an $8 \mathrm{~h}$ period. $\mathbf{a}$, b Prodrug uptake of NM1TFV (green) significantly greater than NM2TFV (orange) and TAF (blue). TAF treatment resulted in the most rapid prodrug conversion to TFV-DP, followed by NM2TFV, and NM1TFV. a, b Values reported are the mean \pm SEM of three replicates. Each experiment was repeated independently three times with equivalent results. c Transmission electron microscopy (TEM) of control, TAF, NM1TFV, and NM2TFV loaded MDM after $8 \mathrm{~h}$ drug incubation with $10 \mu \mathrm{M}$ drug. Scale bar: $2 \mu \mathrm{m}$. For comparison of two groups Student's t-test (two-tailed, unpaired) was used $\left({ }^{\star} P \leq 0.05,{ }^{\star \star} P<0.01,{ }^{\star \star \star} P<0.001,{ }^{\star \star \star \star} P<0.0001\right.$ NM1TFV compared with NM2TFV). A one-way analysis of variance (ANOVA) followed by Tukey's post hoc test was used to compare the prodrug and TFV-DP levels among three treatment groups ( ${ }^{\star} P \leq 0.05$, ${ }^{\star \star} P<0.01,{ }^{\star \star \star} P<0.001,{ }^{\star \star \star \star} P<0.0001$ NM1TFV compared with NM2TFV or TAF). Data were independently reproduced three times. 

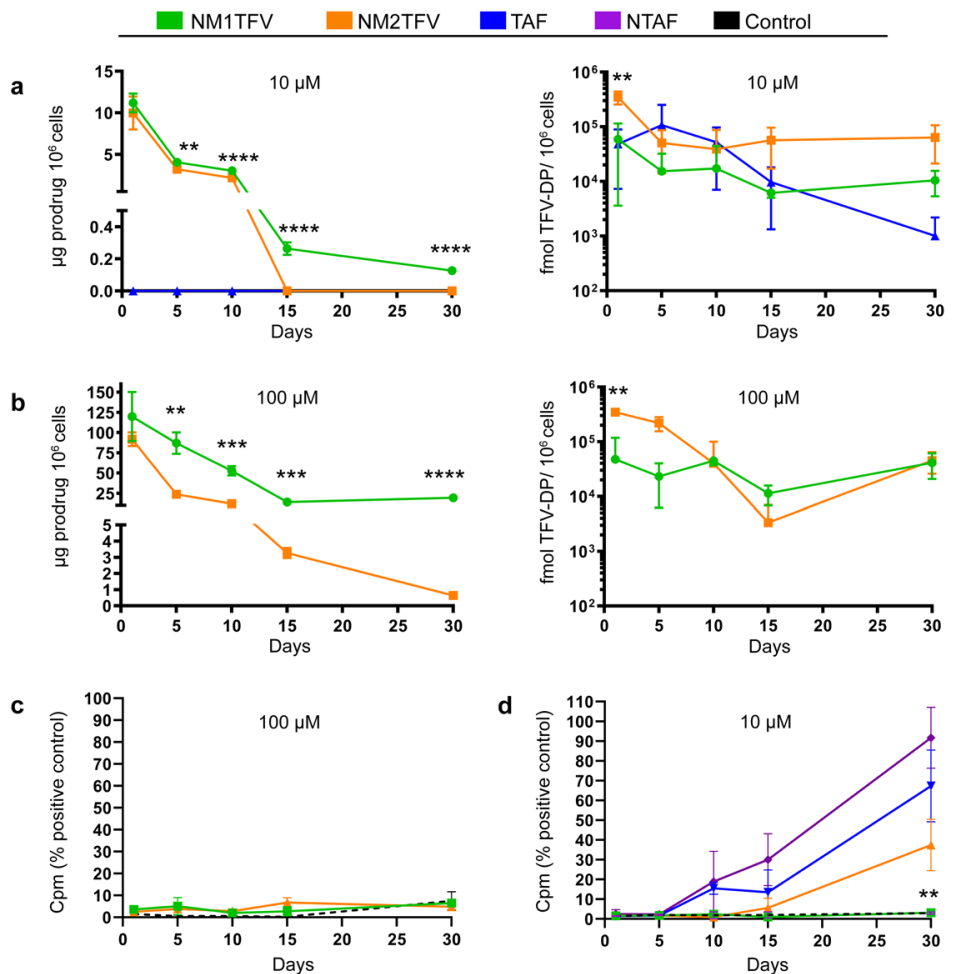
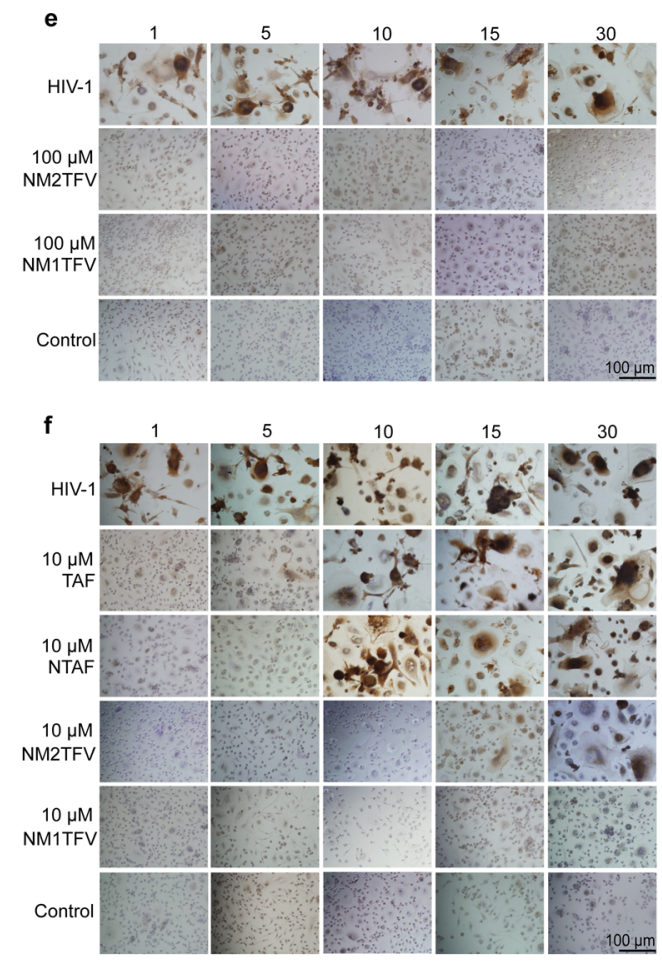

Fig. 4 Long-term cell nanoparticle interactions. Following a $10 \mu \mathrm{M}\left({ }^{\star \star} P=0.0026,{ }^{\star \star \star \star} P<0.0001 \mathrm{NM} 1 \mathrm{TFV}\right.$ vs. NM2TFV prodrug; ${ }^{\star \star} P=0.0096 \mathrm{NM} 1 \mathrm{TFV}$ vs. NM2TFV TFV-DP) or $\mathbf{b} 100 \mu \mathrm{M}$ treatments of NM1TFV (green), NM2TFV (orange) or TAF (blue) ${ }^{\star \star \star} P=0.001511,{ }^{\star \star \star} P=0.000326,{ }^{\star \star \star} P=0.000180$, ${ }^{\star \star \star \star \star} P<0.0001$ NM1TFV vs. NM2TFV prodrug on days 5, 10, 15, and 30, respectively; ${ }^{\star \star} P=0.001911$ NM1TFV vs. NM2TFV TFV-DP), intracellular prodrug (left) and TFV-DP (right) concentrations in MDM were measured over a 30-day period. Drug concentrations are expressed as mean \pm SEM, $N=3$ biological replicates. Following $\mathbf{c}, \mathbf{e} 100 \mu \mathrm{M}$ or $\mathbf{d}, \mathbf{f} 10 \mu \mathrm{M}$ treatments, the antiretroviral activities of NM1TFV and NM2TFV were determined by measures of HIV-1 RT activity in culture supernatants and by cell-associated HIV-1 p24 antigens by immunonocytochemistry. ${ }^{\star \star} P=0.0042$ NM1TFV vs. NM2TFV. e, $\mathbf{f}$ Scale bar: $100 \mu \mathrm{m}$. HIV-1 RT activity is expressed as mean \pm SEM, $N=4$ biological replicates. For comparison of two groups Student's t-test (two-tailed, unpaired) was used $\left({ }^{\star} P \leq 0.05,{ }^{\star \star} P<0.01,{ }^{\star \star \star} P<<0.001,{ }^{\star \star \star \star} P<0.0001\right.$ NM1TFV compared with NM2TFV). A one-way analysis of variance (ANOVA) followed by Tukey's post hoc test was used to compare the prodrug and TFV-DP levels among three treatment groups $\left({ }^{\star} P \leq 0.05,{ }^{\star \star} P<0.01,{ }^{\star \star \star} P<0.001\right.$, $\star \star \star \star * P<0.0001$ NM1TFV compared with NM2TFV).

accumulation of NM1TFV and NM2TFV drug reservoirs in the cytoplasm compared to TAF (Fig. 3c). In CEM-ss T-cells, significantly improved prodrug uptake was observed for NM1TFV compared to NM2TFV and NTAF (Supplementary Fig. 9b). To further confirm the release of ProTides from the nanoparticles and their conversion to active metabolites, we quantified intracellular TFV-DP levels in MDMs after a single exposure to NM1TFV, NM2TFV, or TAF. As shown in Fig. 3a, the $10 \mu \mathrm{M}$ TAF treatment produced the highest TFV-DP concentration, peaking at $8 \mathrm{~h}\left(690,949 \mathrm{fmol} / 10^{6}\right.$ cells). For NM1TFV at $10 \mu \mathrm{M}$, the maximum TFV-DP levels were observed at $8 \mathrm{~h}(7,056.7 \mathrm{fmol} /$ $10^{6}$ cells), and of $57,113 \mathrm{fmol} / 10^{6}$ cells at $8 \mathrm{~h}$ for NM2TFV. Even though lower concentrations of TFV-DP were measured for NM1TFV compared to NM2TFV or TAF, the active metabolite levels were sustained over $8 \mathrm{hrs}$, well above the human $\mathrm{EC}_{90}$ of 40 fmol $/ 10^{6}$ cells. At the $100 \mu \mathrm{M}$ concentration (Fig. 3b), the TFVDP concentrations for NM1TFV and NM2TFV peaked after $8 \mathrm{~h}$, and the difference was not found to be statistically significant $\left(160,867 \mathrm{fmol} / 10^{6}\right.$ cells, and $239,422 \mathrm{fmol} / 10^{6}$ cells, respectively).

Prior data from our laboratories demonstrate sustained and enhanced storage of nanoformulations in $\mathrm{MDM}^{35}$. Thus, retention of TFV nanoformulations in MDM was determined. Following a $10 \mu \mathrm{M}$ treatment, NM1TFV facilitated retention of the prodrug in MDM for up to 30 days $\left(0.13 \mu \mathrm{g} / 10^{6}\right.$ cells), while NM2TFV and native TAF treatments showed no detectable drug within 15 days, and $24 \mathrm{~h}$ respectively (Fig. 4a). At a higher concentration of $100 \mu \mathrm{M}$ (Fig. 4b) both NM1TFV and NM2TFV facilitated prodrug retention for up to 30 days $\left(19.53 \mu \mathrm{g} / 10^{6}\right.$ cells and $0.64 \mu \mathrm{g} / 10^{6}$ cells respectively). To assess the extent of active metabolite retention, intracellular TFV-DP was measured over a 30-day time period after a single exposure of drug for $8 \mathrm{~h}$. Notably, a single treatment of MDMs with NM1TFV or NM2TFV provided steady and sustained TFV-DP levels over the entire 30-day period (Fig. 4a, b). As shown in Fig. 4a, the amount of TFV-DP retained by $10 \mu \mathrm{M}$ NM1TFV-treated MDM was $48,232.4 \mathrm{fmol} / 10^{6}$ cells on day 1 , and $10,507.7 \mathrm{fmol} / 10^{6}$ cells on day 30. Similarly, $10 \mu \mathrm{M}$ of NM2TFV provided sustained TFV-DP concentrations of 349,162 and $64,057.4 \mathrm{fmol} / 10^{6}$ cells at days 1 and 30, respectively (Fig. 4a). TAF demonstrated a rapid decline of the active metabolite concentration compared to NM1TFV or NM2TFV; levels of TFV-DP fell from 59,239.4 fmol/ $10^{6}$ cells at day 1 to $1,013.4 \mathrm{fmol} / 10^{6}$ cells on day 30 . Sustained intracellular TFV-DP concentrations were also observed at the $100 \mu \mathrm{M}$ of NM1TFV or NM2TFV treatments. For NM1TFV, intracellular active metabolite TFV-DP was retained at levels of $47,622.4 \mathrm{fmol} / 10^{6}$ cells at day 1 then $41,219.4 \mathrm{fmol} / 10^{6}$ cells by day 30. Similarly, NM2TFV provided sustained TFV-DP concentrations of 348,132 and $45,144.8 \mathrm{fmol} / 10^{6}$ cells at days 1 and 30, respectively (Fig. $4 \mathrm{~b}$ ). Such sustained-release formulations could maintain effective drug concentrations at cellular and tissue reservoirs of infection. To assess whether enhanced intracellular TFV-DP levels would translate into improved efficacy, MDM were challenged every five days with HIV-1 $1_{\mathrm{ADA}}$ to an end point of 30 days following an $8 \mathrm{~h}$ drug loading and assayed quantitatively 
for HIV-1 RT activity, as well as qualitatively for HIV-1 p24 antigen expression (Fig. $4 \mathrm{c}-\mathrm{f}$ ). At the $100 \mu \mathrm{M}$ dose, no statistically significant differences in RT activity were detected in media from NM1TFV and NM2TFV-treated cells (Fig. 4c). At $10 \mu \mathrm{M}$, significantly lower RT activity was detected in media from NM1TFV and NM2TFV-treated cells compared to TAF and NTAF treatment beginning on day 10 post-treatment and continuing out to 30 days (Fig. 4d). NM1TFV treatment also resulted in significantly lower RT activity compared to NM2TFV at day 30 (Fig. 4c, d). Both 10 and $100 \mu \mathrm{M}$ NM1TFV suppressed HIV-1 RT activity by $>92 \%$ and $>96 \%$ at all time points, respectively (Fig. 4c, d). While $100 \mu \mathrm{M}$ NM2TFV also suppressed HIV-1 RT activity by $>92 \%$ at all the time points, treatment with $10 \mu \mathrm{M}$ of NM2TFV provided viral inhibition of $62.57 \%$ at day 30 . Similarly, $10 \mu \mathrm{M}$ NTAF and TAF provided viral inhibition of 81.1 and $84.5 \%$ respectively at day 10 , with complete viral breakthrough occurring at day 30 (Fig. 4d, f). In all, these findings demonstrate that NM1TFV improves intracellular delivery of TFV-DP with enhanced drug potency and sustained antiretroviral efficacy.

PK testing. To assess the $\mathrm{PK}$ and drug biodistribution (BD) profiles, male and female Sprague-Dawley rats were injected intramuscularly (IM) with a single dose of $75 \mathrm{mg} / \mathrm{kg}$ body weight TFV-equivalents of NM1TFV, NM2TFV, or NTAF. The area under the curve (AUC) for whole blood prodrug (6,427 (ng* days)/mL) in NM1TFV treated animals was found to be statistically higher than whole blood prodrug $(2,792$ (ng* days)/mL) in NM2TFV animals (GraphPad Prism v9.0.0.0 software, one-way ANOVA, $P=0.0340$ ) (Fig. 5a). Additionally, compared to NM2TFV, NM1TFV resulted in significantly greater whole blood prodrug concentrations on days 14 and 42 (Fig. 5a). Notably, NM1TFV treatment resulted in significantly higher TFV concentrations in whole blood compared to NTAF on days 14, 28, and 56 ( $p<0.0001, p=0.0326, p=0.024$, respectively). However, no statistically significant differences in whole blood TFV levels were observed between NM1TFV and NM2TFV treatment groups (Fig. 5a). NM1TFV significantly improved tissue drug delivery compared to NM2TFV and NTAF. Specifically, compared to NTAF and NM2TFV, NM1TFV provided significantly higher TFV concentrations in spleen, lymph nodes, liver, gut, kidneys, and site of injection at 28 and 56 days-post drug administration (Fig. 5b-f and Supplementary Fig. 10). For NM1TFV at day 56, the TFV levels were 102.4, 26, 12.5, 12.5, $1,258.1$, and $8,883.2 \mathrm{ng} / \mathrm{g}$ tissue in the liver, spleen, lymph nodes, gut, kidney, and site of injection, respectively. By comparison, TFV levels 56 days after NTAF treatment were 0.1, 1.8, 1.03, 5.58, and $18.8 \mathrm{ng} / \mathrm{g}$ tissue in liver, lymph nodes, gut, kidneys, and site of injection, respectively. Similarly, TFV levels at day 56 after NM2TFV treatment were $4.87,1.58,0.1,20.28$, and $193.98 \mathrm{ng} / \mathrm{g}$ tissue in the liver, spleen, gut, kidney, and site of injection, respectively. Also, NM1TFV displayed substantially higher prodrug levels compared to other treatments (Fig. 5b-f and Supplementary Fig. 10). For NM1TFV at day 56, the prodrug levels were $65.6,151.3,27.8,18.3,5.3$, and $433,475 \mathrm{ng} / \mathrm{g}$ tissue in the liver, spleen, lymph nodes, gut, kidney, and site of injection, respectively (Fig. 5b-f, Supplementary Fig. 10b). The data supports the contention that NM1TFV undergoes slow nanoparticle dissolution and prodrug hydrolysis in vivo compared to NM2TFV and NTAF. At the site of injection, significantly greater levels of prodrug were observed for NM1TFV at day 28 $(1,253,500 \mathrm{ng} / \mathrm{g}$ tissue) and 56 (433,475 ng/g tissue) (Fig. 5f). Based on the initial dose, the total amount of drug at the site of injection after NM1TFV injection was $10 \%$ at day 28 and $5 \%$ at day 56 (Supplementary Fig. 11b). For NM2TFV, the muscle drug depot was $0.1 \%$ and $0.05 \%$ of the initial dose at days 28 and 56 (Supplementary Fig. 11b). The molar ratios of the prodrug to TFV levels in the muscle demonstrated that a significant proportion of drug after NM1TFV treatment remained in prodrug form when compared against NM2TFV (Supplementary Fig. 11c). Additionally, Pearson correlation determined that prodrug concentration at the site of an injection directly correlated with prodrug and parent drug concentration in most of the tissues, supporting a linear relationship between the magnitude of the prodrug intramuscular depot and tissue drug concentrations (two-tailed, $\alpha=0.05$, Supplementary Fig. 11d). In all, the data suggest that NM1TFV is more stable at the site of injection and is absorbed more slowly than NM2TFV from the intramuscular depot. Given the enhanced stability of the M1TFV prodrug in the muscle at day 56, NM1TFV offers the potential for sustained drug delivery beyond two months.

TFV-DP measurements. We next determined the concentration of the active metabolite in key HIV and hepatitis B virus (HBV) target cells and tissues (Fig. 6). In PBMCs, a single treatment with either NM1TFV, NM2TFV, or NTAF demonstrated TFV-DP concentrations above the human $\mathrm{EC}_{90}\left(40 \mathrm{fmol} / 10^{6}\right.$ cells $)$ during the entire study duration (Fig. 6a). Specifically, TFV-DP levels for NM1TFV, NM2TFV, and NTAF at 8 weeks were 156.28, 291.75, and $305.33 \mathrm{fmol} / 10^{6}$ cells, respectively. Similarly, no significant differences in TFV-DP levels were recorded in splenoctyes, nonparenchymal liver cells, and in vaginal tissue among the three treatments (Fig. 6). However, NM2TFV resulted in significantly higher TFV-DP levels in lymphocytes at D28 (239.319 fmol/106 cells), compared to NM1TFV (156.26 fmol/106 cells) and NTAF (71.94 fmol/106 cells) (Fig. 6b). Compared to NM2TFV and NTAF, NM1TFV provided significantly higher TFV-DP levels in parenchymal liver cells at D56 $\left(328.02 \mathrm{fmol} / 10^{6}\right.$ cells $)$ and in rectal tissue at all time points $\left(36,575.61 \mathrm{fmol} / 10^{6}\right.$ cells on D28, and $11,276.31 \mathrm{fmol} / 10^{6}$ cells on D56, Fig. $6 \mathrm{~d}, \mathrm{f}$ ). Importantly, at the end of the study, NM1TFV exhibited sustained PBMC and tissue TFV-DP concentrations above the level associated with effective pre-exposure prophylaxis in humans. Given the high muscle M1TFV levels at day 56, it is feasible to achieve sustained therapeutic TFV-DP levels beyond 2 months.

Toxicology and evaluation of injection site. Toxicity was assessed in SD rats after NM1TFV, NM2TFV, and NTAF treatment. Animal weights were recorded weekly and organ to body weight ratios were determined on the days of sacrifice (days 28 and 56); plasma and tissues were collected for hematologic, chemistry, and histopathology (Fig. 7 and Supplementary Figs. 12-14). Controls were age and sex-matched and evaluated in replicate untreated rats. Erythema and edema were not observed at the site of injection in any of the treatment groups. No statistically significant differences in body weights were observed between the treatment groups (Supplementary Fig. 13b). Transient differences in lung and spleen to body weight ratios were observed between NM1TFV and NTAF groups at day 28. However, no statistically significant differences in organ to body weight ratios were detected on day 56 (Supplementary Fig. 13a). No hematological abnormalities were detected (Supplementary Fig. 13c). There were no notable differences between treatment groups for comprehensive chemistry profiles (Supplementary Fig. 12), suggesting that NM1-, NM2- TFV and NTAF did not adversely affect organ function. Hematoxylin and eosin-stained tissue sections, examined by a certified pathologist in a blinded fashion, revealed no abnormal pathology in the kidney and livers of NM1TFV and NTAF treated animals (Supplementary Fig. 14b). Injection site reactions were examined following 

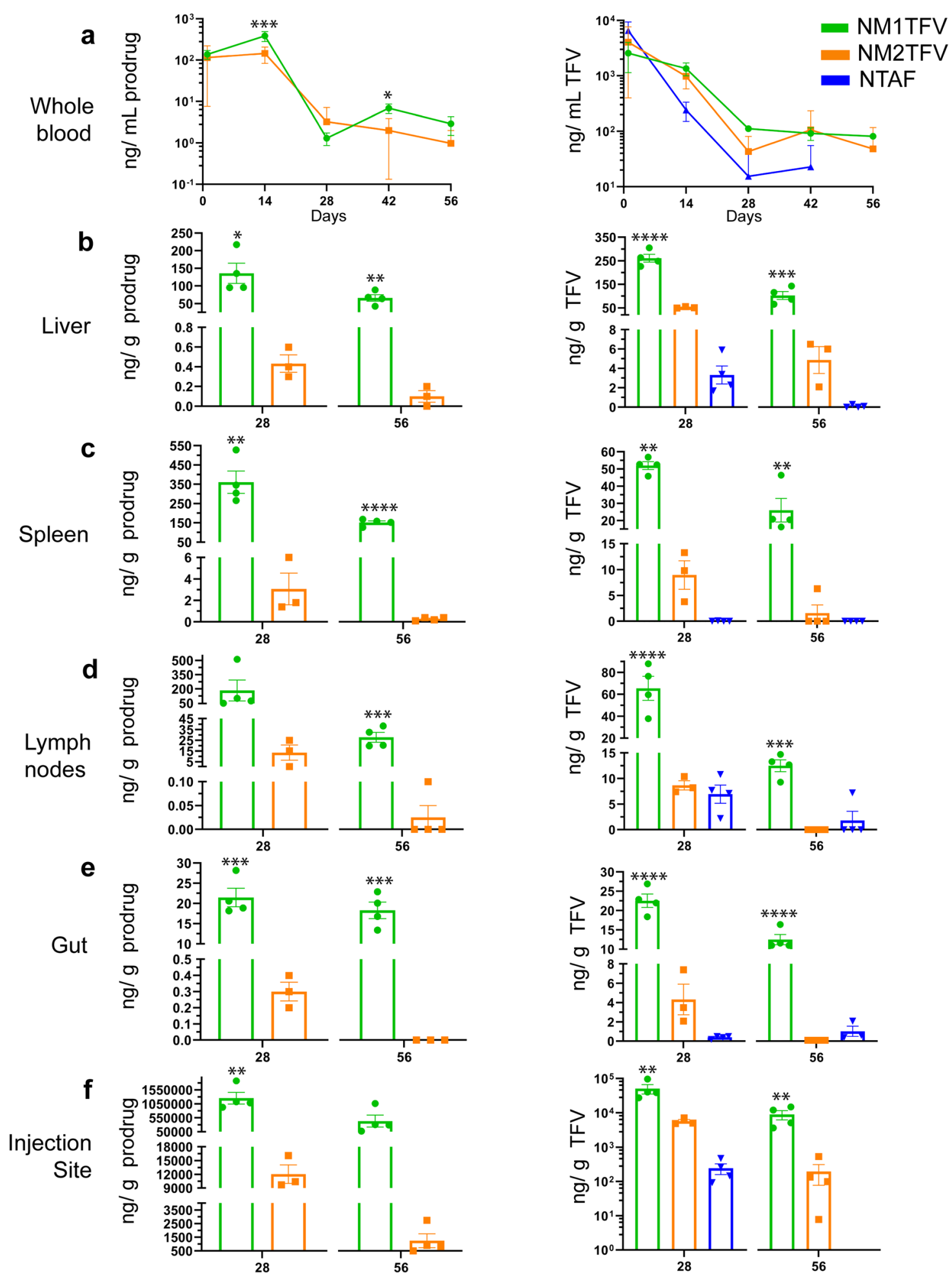

Fig. 5 Pharmacokinetics. SD rats were administered a single IM dose of NM1TFV (green), NM2TFV (orange), or NTAF (blue) (75 mg/kg TFV-eq.) to determine pharmacokinetic (PK) profiles. a Prodrug (left) and TFV (right) concentrations in blood were determined at days 1 and 14 then every two weeks for 8 weeks $\left({ }^{\star \star \star} P=0.0001,{ }^{\star} P=0.0177\right.$ NM1TFV vs. NM2TFV prodrug). Tissue biodistribution of M1TFV, M2TFV, and TFV was assessed at 28 and 56 days after injection in the $\mathbf{b}$ liver $\left({ }^{\star} P=0.0107,{ }^{\star \star} P=0.002\right.$ NM1TFV vs. NM2TFV prodrug; ${ }^{\star \star \star \star} P<0.0001,{ }^{\star \star \star} P=0.0007$ NM1TFV vs. NM2TFV TFV), c spleen $\left({ }^{\star \star} P=0.0034,{ }^{\star \star \star \star} P<0.0001 \mathrm{NM1TFV}\right.$ vs. NM2TFV prodrug; ${ }^{\star \star} P=0.004,{ }^{\star \star} P=0.0055, \mathrm{NM} 1 \mathrm{TFV}$ vs. NM2TFV TFV), d lymph node ${ }^{\star \star \star \star} P=0.0009$ NM1TFV vs. NM2TFV prodrug; ${ }^{\star \star \star} P=0.0001,{ }^{\star \star \star \star} P<0.0001$ NM1TFV vs. NM2TFV TFV), e gut $\left({ }^{\star \star \star} P=0.0006,{ }^{\star \star \star} P=0.0007\right.$ NM1TFV vs. NM2TFV prodrug; ${ }^{\star \star \star \star} P<0.0001$ NM1TFV vs. NM2TFV TFV), and $\mathbf{f}$ site of injection $\left({ }^{\star \star} P=0.0043 \mathrm{NM} 1 \mathrm{TFV}\right.$ vs. NM2TFV prodrug; ${ }^{\star \star} P=0.0084$, ${ }^{\star \star} P=0.0085$ NM1TFV vs. NM2TFV TFV). Data are expressed as mean \pm SEM where $N=4$ biological replicates. Prodrug concentrations were compared using two-tailed unpaired $t$-test $\left({ }^{\star \star \star \star} P<.0001,{ }^{\star \star \star} P<.001,{ }^{\star \star} P<.01,{ }^{\star} P<.05\right.$ NM1TFV compared with NM2TFV). To compare TFV levels between three or more groups, ordinary one-way ANOVA followed by Tukey's post hoc test with a single pooled variance was used $\left({ }^{\star \star \star \star} P<.0001,{ }^{\star \star \star} P<.001,{ }^{\star \star} P<.01\right.$, ${ }^{\star} P<.05$ NM1TFV compared with NM2TFV). 
a
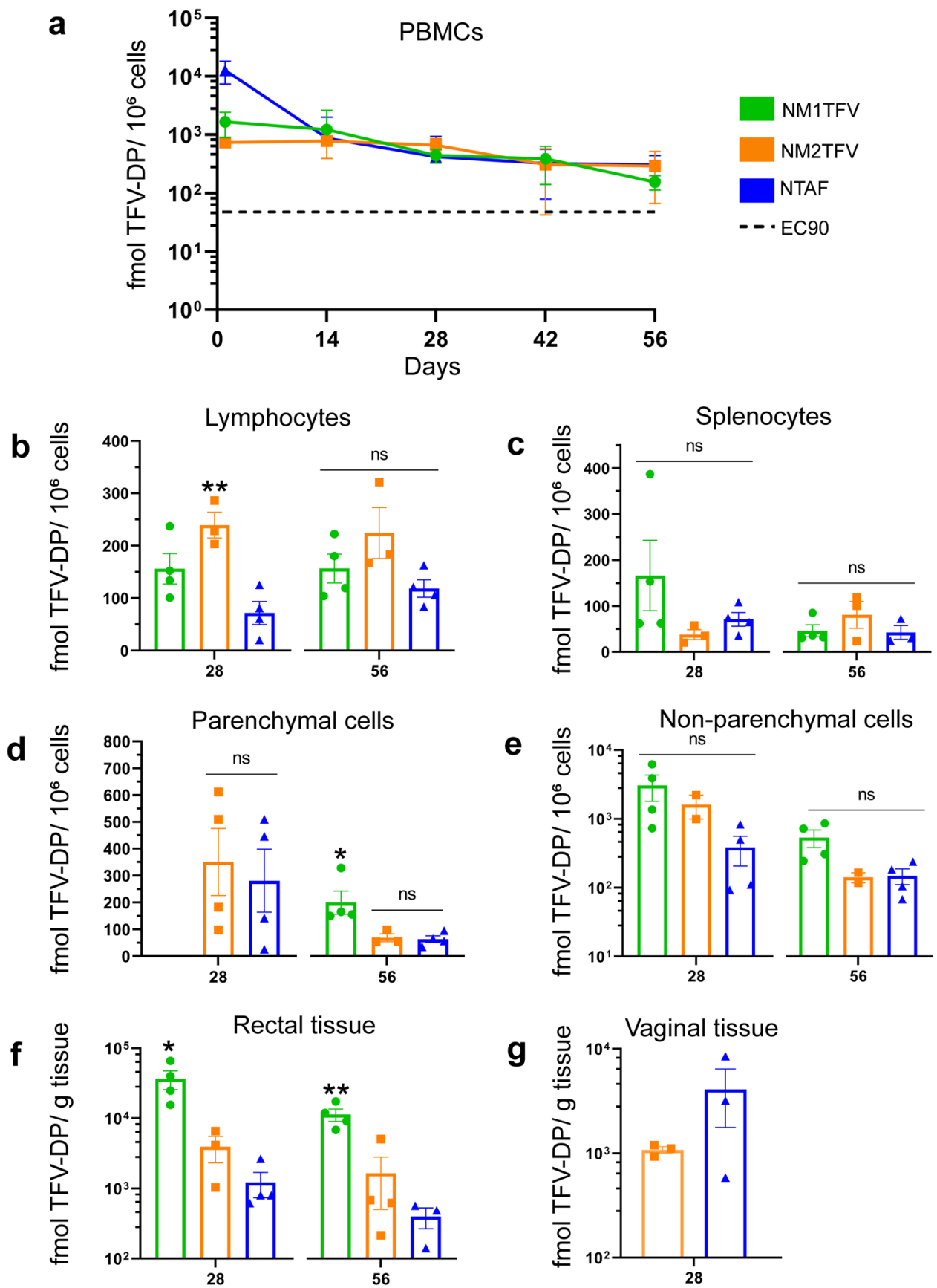

Fig. 6 In vivo TFV-DP Measurements. SD rats were administered a single IM dose of NM1TFV (green), NM2TFV (orange), or NTAF (blue) (75 mg/kg TFV-eq.) to determine pharmacokinetic (PK) profiles. a TFV-DP concentrations in PBMCs were determined at days 1 and 14 then every two weeks for 8 weeks. Tissue biodistribution of TFV-DP was assessed at 28 and 56 days after injection in the $\mathbf{b}$ lymphocytes ( ${ }^{\star \star} P=0.0024, N M 2 T F V$ vs. NTAF), $\mathbf{c}$ splenocytes, d parenchymal liver cells $\left({ }^{\star} P=0.0372, \mathrm{NM} 1 \mathrm{TFV}\right.$ vs. NM2TFV), e non-parenchymal liver cells, $\mathbf{f}$ rectal tissue $\left({ }^{\star} P=0.0115,{ }^{\star \star} P=0.0058\right.$ NM1TFV vs. NM2TFV), and $\mathbf{g}$ vaginal tissue. Data are expressed as mean \pm SEM where $N=4$ biological replicates. Vaginal TFV-DP concentrations were compared using a two-tailed unpaired $t$-test $\left({ }^{\star \star \star \star} P<.0001,{ }^{\star \star \star} P<.001,{ }^{\star \star} P<.01,{ }^{\star} P<.05\right.$ NM1TFV compared with NM2TFV). a-f To compare TFV-DP levels between three or more groups, ordinary one-way ANOVA followed by Tukey's post hoc test with a single pooled variance ${ }^{\star \star \star \star} P<.0001,{ }^{\star \star \star} P<.001$, ${ }^{\star \star} P<.01,{ }^{\star} P<.05$ NM1TFV compared with NM2TFV).

NM1TFV and NTAF injections showing minimal granulomatous reactions (Fig. 7 and Supplementary Fig. 14a). Specifically, one week after NM1TFV administration, amorphous material, a central area containing foamy macrophages and cellular debris were present at the injection site, typical of necrosis. Spindleshaped fibroblasts and vascular proliferation surrounded the necrotic region, indicating wound healing and granulation tissue formation. Histiocytic infiltration of the intramuscular depot was also observed, including minimal infiltration of the adjacent skeletal muscle. Regions of extracellular amorphous eosinophilic material were also noted. Interspersed within the nuclear debris and foamy histiocytes were small, spherical basophilic granules, mostly extracellular. Given that NM1TFV carries a negative surface charge (Fig. 2a) and is readily stained by hematoxylin (Fig. 7a), the observed basophilic granules were recorded as NM1TFV nanoparticle aggregates. Clusters of these basophilic 

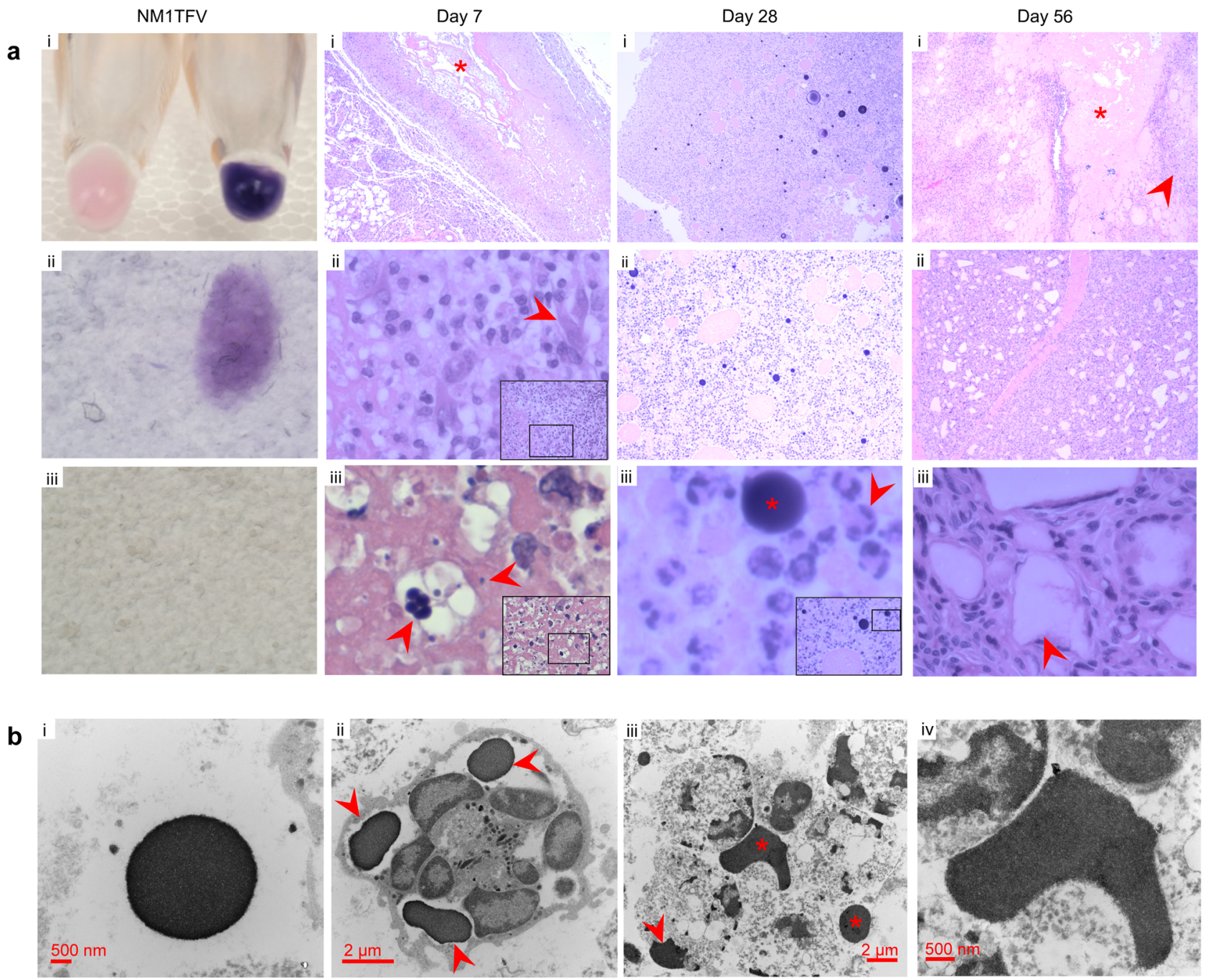

Fig. 7 Injection site histopathology. Following a single IM dose of NM1TFV or NTAF male SD rats were sacrificed on days 7, 28, and 56. a H\&E staining of NM1TFV (i) revealed the formulation was readily stained by hematoxylin (ii), but not eosin (iii). For the histological examination of the site of injection, $5 \mu \mathrm{M}$ sections of paraffin-embedded tissues were stained with hematoxylin and eosin. Images were captured using a Nuance EX multispectral imaging system affixed to a Nikon Eclipse E800 microscope (Nikon Instruments). At day 7, a necrotic focus was observed ( $\mathrm{i}$, indicated by ${ }^{*}$ ), surrounded by histiocytes, and fibroblasts (ii, arrow). Clusters of foreign material, likely NM1TFV were also observed interspersed in nuclear debris (iii, arrows). By day 28, larger extracellular basophilic staining globules (iii, indicated by *) along with small basophilic intracellular granules present in the histiocytes (iii, indicated by arrow), along with foreign body granulomatous reaction. At 56 days, the foreign material appeared mostly amorphous ( $i$, indicated by ${ }^{*}$, with a foreign body giant cell granulomatous reaction (ii, iii). b TEM images of the NM1TFV site of injection at day 28 (i \& ii), and 56 (iii \& iv) confirmed the light microscopic findings. a, b Study was performed once; images are representative of the histological findings from three biological replicates. Magnification of each micrograph is as follows: $1 \times, 60 \times, 60 \times$ for NM1TFV i, ii, iii, respectively. 4×, 20x, 40× for Day 7 i, ii, iii, respectively. 4×, 10×, 40× for Day 28 i, ii, iii,

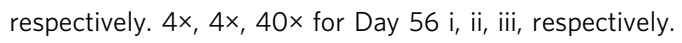

granules were noted 7 days following the intramuscular injection of the prodrug nanoformulation, suggesting aggregation of NM1TFV particles (Fig. 7a). By day 28, there were large extracellular basophilic staining globules along with small basophilic intracellular granules present in the histiocytes, indicating further aggregation of NM1TFV (Fig. 7a). This was accompanied by a foreign body granulomatous reaction, including foreign body giant cells (Fig. 7a). When sections were imaged by TEM, the basophilic granules observed by light microscopy appeared as non-cellular electron-dense aggregates, further suggesting that this foreign material was the NM1TFV formulation and corresponding to the presence of the prodrug by chemical analysis. These electron-dense aggregates of NM1TFV appeared extracellularly and intracellularly within the histiocytes (Fig. 7b). At
56 days, the foreign material appeared mostly amorphous, with a foreign body giant cell granulomatous reaction. Extracellular basophilic globules and finer granular intracellular basophilic material were present, indicating sustained phagocytosis of NM1TFV by histiocytes (Fig. 7). One rat at 56 days showed a small focus of fibrosis. TEM observations supported the light microscopic findings, showing extracellular electron-dense aggregates of NM1TFV along with intracellular aggregates within the histiocytes (Fig. 7b). The injection sites in rats treated with NTAF showed small areas of histiocytic infiltrate at 28 days, mostly in the interstitium (Supplementary Fig. 14a). Amorphous foreign material was present, and this persisted in one of the three rats at 56 days, including a foreign body giant cell granulomatous reaction (Supplementary Fig. 14a). There was no evidence of 
muscle degeneration, basophils or eosinophils, cellulitis, or abscesses in any of the treatment groups at any time point examined.

\section{Discussion}

Although LA ARV formulations could impact HIV treatment and prevention, the physicochemical properties of current oral ARVs are not compatible with existing sustained release formulation approaches. Thus, improvements in drug regimens are emergently needed for PrEP and maintenance therapies. While current HIV treatment consists of three-drug regimens, and current PrEP consists of a two-drug regimen; both require once-daily dosing. Change in these paradigms is now possible. This resides in harnessing the potential role of TAF in LA delivery systems. Indeed, TAF and TDF prodrugs demonstrate potent activity against HIV and $\mathrm{HBV}$ infections making the agent the most used antiretroviral ${ }^{36,37}$. The combination of TFV and emtricitabine is effective at preventing HIV-1 transmission when taken daily ${ }^{38,39}$. Additionally, the current recommended first-line antiretroviral regimen for HIV treatment consists of combinations of TAF/TDF and emtricitabine together with an integrase, protease, or nonnucleoside reverse transcriptase inhibitor ${ }^{40}$. Despite its effectiveness, the need for strict regimen adherence, noted toxicities, and restricted tissue penetrance all highlight immediate needs for improved formulation strategies ${ }^{41-43}$. Thus, LASER ART and delivery devices for ARVs are being developed to extend dosing intervals, reduce systemic toxicity, and improve PK profiles $4,16,21,44-46$. Herein, we report on the development of TFV ProTides with improved prodrug stability. In this approach, the ionizable phosphate groups in the drug are masked by aromatic and amino acid ester moieties, which upon delivery undergo intracellular enzymatic or chemical hydrolysis to release the free form of the phosphorylated compounds for subsequent activation and competitive inhibition of the viral reverse transcriptase. The lead TFV ProTide candidate (M1TFV) facilitated the production of stable aqueous nanocrystals that exhibited enhanced drug and active metabolite delivery into macrophages and CEM-ss CD4+ $\mathrm{T}$ cells. PK and efficacy evaluations showed significant improvements in drug apparent half-life, biodistribution, and antiretroviral activities over TAF. These data, taken together, support the idea that NM1TFV could enable sustained release of TFV-DP to enable dosing intervals for TFV beyond every two months.

TAF is a ProTide of the acyclic nucleoside phosphonate drug tenofovir. The application of ProTide technology to TFV led to the discovery of TAF that is characterized by enhanced potency, improved lymphoid tissue drug delivery, and has also limited the risk for nephrotoxicity and loss of bone mineral density associated with another prodrug of tenofovir, tenofovir disoproxil fumarate $(\mathrm{TDF})^{18,19}$. Despite the advantages conferred by TAF, its application in LA systems remains limited by the inherent hydrolytic instability of the prodrug. The enhanced potency and chemical composition of TAF further limit the possibility of making direct chemical modifications to the prodrug to improve compound stability without affecting efficacy and safety profiles. Moreover, prior attempts to improve prolonged TAF stability by either using excipients, formulation approaches, or $\mathrm{pH}$ modulation are yet to demonstrate successful in vivo outcomes ${ }^{22,45}$. To these ends, we explored TFV ProTides bearing safe but bulky hydrophobic lipids not explored by conventional ProTide designs due to their presumed undesired effect on compound dissolution properties in physiological fluids to affect efficacy. Two hydrophobic and lipophilic stable docosyl phenylalanyl and alanyl ester TFV ProTides (M1TFV, M2TFV) were created and nanoformulated by high-pressure homogenization as aqueous suspensions of crystalline drugs stabilized by P407 surfactant. Even though solubility and dissolution rates are inherent properties of organic compounds, the actual dissolution rate is dependent on the surface area and degree of saturation. The reduced particle size was expected to enhance prodrug dissolution to produce rapid absorption and therapeutic onset upon administration. Since solubilized form of TAF is $\mathrm{pH}$ and water-sensitive, saturated aqueous solid drug nanoparticles were prepared at a $\mathrm{pH}$ of 5.5 to maintain prodrug stability within the formulation. The recorded uniform particle sizes, narrow polydispersity indices, and negative zeta potentials for NM1TFV and NM2TFV formulations are suggestive of nanoparticle stability and homogeneity. Importantly, the high drug loading capacity for the TFV ProTide nanocrystals could facilitate reduced injection volumes.

After producing and characterizing the stable TFV ProTide formulations, we then evaluated their abilities to enter and slowly release the drug within HIV-1 target cells. Nanocrystal delivery to phagocytic and highly mobile macrophages facilitates drug transfer to $\mathrm{CD} 4+\mathrm{T}$ cells and other restricted tissue reservoirs of infection $^{12}$. We have previously demonstrated that once inside macrophages, drug nanocrystals are stored in the late- and recycling-endosomes ${ }^{35}$. This intracellular particle storage protects drugs from systemic metabolism and prolongs half-life, ultimately leading to improved $\mathrm{PK}$ and drug $\mathrm{BD}$ profiles ${ }^{11,12}$. Also, the transformation of ProTides into active nucleotide metabolites to competitively inhibit the activity of HIV reverse transcriptase is mediated by intracellular kinases, underscoring the importance of cellular NRTI delivery. Compared to NTAF and NM2TFV, treatment of human MDM with NM1TFV resulted in enhanced cellular uptake and intracytoplasmic retention of the prodrug and TFV-DP over 30 days. The observed intracellular TFV-DP levels for NTAF and NM2TFV were expected given their rapid hydrolysis rates when compared to NM1TFV. Prior studies have also shown that TFV-DP exhibits an intracellular half-life of 60-100 $\mathrm{h}^{4}$ and is likely to be extended when delivered in form of solid drug nanoparticles that readily undergo dissolution prior to prodrug release, hydrolysis, and activation. Consequently, NM1TFV exhibited sustained antiretroviral activity as determined by HIV-1p24 antigen expression and RT activity. Together, these characterization data sets demonstrate that NM1TFV improves prodrug stability, cell uptake, retention, and efficacy.

Optimal approaches for effective treatment and prevention of HIV-1 infection should deliver and sustain therapeutic drug concentrations at cellular and tissue sites of infection ${ }^{47}$. We therefore investigated the abilities of NTAF, NM1TFV, and NM2TFV to improve drug PK and biodistribution. Pharmacokinetic tests in SD rats demonstrated TFV-DP levels in PBMCs at or above $100 \mathrm{fmol} /$ million cells for 56 days for all treatments. Notably, NM1TFV exhibited sustained intramuscular and enhanced lymphatic tissue drug levels compared to NM2TFV and NTAF. Toxicological assessments (CBC, comprehensive serum chemistry profiles, organ to body weight ratios, kidney and liver histopathology) demonstrated that neither NM1TFV, NM2TFV, nor NTAF induced systemic or organ-specific adverse effects. Following intramuscular administration of nanoformulations, the injection site serves as a primary depot from where the drug is slowly absorbed and eventually released into circulation (Figs. 5, 7 and Supplementary Fig. 10). Though, defining the precise mechanisms of distribution of the intramuscular drug nanosuspensions represents a current knowledge gap. Prior studies have shown that absorption of solid drug nanocrystals following intramuscular administration is dependent on particle size and rate of dissolution, host protein adsorption on the particle surface, and type of primary depot formed (particle agglomerates or dispersion $)^{48}$. In these prior studies, the local inflammatory response was found to affect nanoparticle phagocytosis efficiency, prodrug stability, and uptake of lipophilic compounds by the 
lymphatic system ${ }^{48-52}$. For TFV prodrugs, minimizing extracellular prodrug hydrolysis at the site of injection would limit the accumulation of polar and ionic TFV or ProTide degradation products in the muscle. Compared to TAF prodrug, earlier studies have demonstrated direct links between extracellular TFV and delayed wound healing, marked alterations in epidermal cell proteomics and ulcerations ${ }^{53,54}$, underscoring the importance of prodrug stability in extracellular compartments. Of particular note, NM1TFV demonstrated sustained prodrug stability at the site of injection.

Intramuscular injection of NM1TFV nanocrystals induced typical, expected subclinical granulomatous foreign body reactions ${ }^{55}$. The observed granuloma formation and subsequent intracellular sequestration of NM1TFV by infiltrating histiocytes and giant cells facilitate drug uptake and accumulation within reticuloendothelial and lymphoid tissues to create secondary drug depots (Figs. 5, 6e, 7). Inflammatory granulomatous tissue reactions have previously been observed for similar FDAapproved long-long-acting injectable aqueous drug nanosuspesions ${ }^{48-52}$ where macrophage infiltration and subsequent phagocytosis of nanocrystals is known to modulate biphasic flip-flop pharmacokinetics by promoting both prodrug dissolution and activation ${ }^{48,50}$. Similarly, injection site analysis by TEM demonstrated that the reticuloendothelial system and immune cell infiltration play important roles in lipophilic drug nanocrystal distribution into tissues ${ }^{56}$. Therefore, in addition to the inherent physicochemical properties of the active pharmaceutical ingredient and nanoparticle, local tissue responses likely play a considerable role in the enhanced drug biodistribution to the reticuloendothelial system and the sustained release profile of NM1TFV. Importantly, intramuscularly delivered NM1TFV did not exhibit severe injection site reactions such as allergic reactions, cellulitis, abscesses, or chronic necrosis, the latter of which has been associated with subcutaneous TAF implants ${ }^{57}$. In addition to improved PK and safety profiles, in proof-of-concept pharmacodynamics studies NM1TFV demonstrated antiviral efficacy in $\mathrm{HBV}$-infected humanized mice ${ }^{58}$. These results are a first step towards producing a long-acting TFV for human use by affecting cell and tissue drug penetration, and antiretroviral activity, and drug apparent half-life.

Long-acting NM1TFV can potentially extend antiretroviral drug administration from daily to beyond bimonthly. The TFV ProTides reported in this work confer therapeutic and safety benefits over conventional ProTides or other previously published long-acting TFV formulations. For instance, a previous report demonstrated that TFV-DP levels in PBMCs of humanized mice declined below $100 \mathrm{fmol} / 10^{6}$ cells less than a week after subcutaneous administration of $200 \mathrm{mg} / \mathrm{kg}$ TAF loaded nanoparticles ${ }^{25}$. In contrast, our ProTide formulations sustained TFV-DP levels above this level in PBMCs for 2 months using a dose 2.7 times lower than what was previously reported ${ }^{25}$. Therefore, these developed formulations may facilitate the reduction of injection volumes without compromising therapeutic efficacy. Another technology being explored for sustained release of TFV is implants ${ }^{21,44-46}$. Implantable devices containing TAF are of increased interest due to the drug's potency and long half-life of the active metabolite 22,59 . However, a recent report on subcutaneous TAF implants inserted in rabbits and rhesus macaques demonstrated substantial histological changes in tissues surrounding the implant site ${ }^{57}$. Significant tissue necrosis was noted after 5 weeks and worsened by 12 weeks in animals implanted with TAF-containing devices ${ }^{57}$. Though a necrotic focus was observed one week after prodrug formulation injection, in contrast to what had been reported for TAF implants, no adverse histological changes were observed at the site of injection for the 2-month time period of the study. This observation is linked, in part, to the sustained prodrug and formulation stability within the muscle. With injectables, one potential drawback includes the inability to remove the drug in the case of adverse events. Utilizing an oral lead to preemptively identify adverse drug reactions can minimize the risk of such events. Additionally, in the case of NM1TFV, the predominant drug depot at the site of injection could offer the possibility of surgical removal in emergent cases. Thus, NM1TFV could potentially overcome major obstacles associated with the transformation of TFV into longacting products.

In the present study, NM1TFV significantly improved drug and TFV-DP biodistribution compared to NM2TFV and NTAF. These results underscore the importance of extended PK evaluations beyond two months to elucidate potential clinical dosing intervals. To this end, future studies will involve 6-month PK studies of NM1TFV in rats and rhesus macaques. Given recent reports from HIV Prevention Trials Network (HPTN) 083 and HPTN 084 studies demonstrating that long-acting injectable cabotegravir (CAB-LA) administered every two months is more effective than daily pills in preventing HIV acquisition ${ }^{60}$, it is clear that long-acting formulations may represent an important tool in controlling the HIV epidemic. Given the important role of TFV prodrugs in combination therapies, the lead candidate NM1TFV could have a major impact on HIV treatment and prevention. TFV also inhibits major co-infections, such as $\mathrm{HBV}^{61}$. In vivo proof of concept studies to assess the efficacy of NM1TFV has demonstrated the ability of the formulation to suppress HBV replication in humanized mice over 4 weeks following a single intramuscular dose $\mathrm{e}^{58}$. Future studies will examine the use of NM1TFV for both monotherapy for HBV infection, and for treatment of HIV-HBV coinfections.

In summary, two lipophilic aqueous stable TFV ProTide nanoformulations, NM1TFV and NM2TFV, were created to enhance intracellular drug delivery and retention. The lead NM1TFV formulation demonstrates enhanced and sustained PBMC and tissue TFV-DP levels four times higher than the $\mathrm{EC}_{90}$ for two months after a single intramuscular drug administration into Sprague Dawley rats. NM1TFV provides an attractive candidate for beyond every two months dosing of TFV to improve treatment outcomes.

\section{Methods}

Monophenyl tenofovir was purchased from BOC Sciences (Shirley, NY). TAF hemifumarate was a generous gift from Gilead Sciences Inc. (Foster City, CA). N(carbobenzyloxy)- L-phenylalanine, $\mathrm{N}$-(carbobenzyloxy)- L-alanine, dicholoromethane (DCM), diethyl ether, anhydrous methanol (MeOH), anhydrous chloroform, anhydrous tetrahydrofuran (THF), anhydrous acetonitrile (ACN), anhydrous $\mathrm{N}, \mathrm{N}$-dimethyl formamide (DMF), 1-octanol, imidazole, triethylamine, 1-docosanol, triethyl silane $\left(\mathrm{Et}_{3} \mathrm{Si}\right)$, thionyl chloride, pluronic F127 (P407), polyethylene glycol 3350, ciprofloxacin, 3-(4,5-dimethylthiazol-2-yl)-2,5-diphenyltetrazolium bromide (MTT), dimethyl sulfoxide (DMSO) ammonium bicarbonate, HEPES buffer solution, potassium chloride, ciprofloxacin, paraformaldehyde (PFA) and 3,3'-diaminobenzidine were purchased from Sigma-Aldrich (St. Louis, MO). Palladium, $10 \%$ on activated carbon, was purchased from STREM Inc. (Newburyport, MA). 1-[Bis(dimethylamino)methylene]-1H-1,2,3-triazolo[4,5-b] pyridinium 3-oxidhexafluorophosphate (HATU) was obtained from Chem Impex Intl Inc. (Wood Dale, IL), Optima MS-grade MeOH, HPLC-grade acetonitrile (MeOH), Optima MS-grade water, cell-culture-grade water (endotoxin-free), and $10 \mathrm{mg} / \mathrm{mL}$ gentamicin reagents were purchased from Fisher Scientific (Hampton, NH). Monoclonal mouse anti-human HIV-1 p24 (clone Kal-1, 1:200 dilution) and polymer-based horseradish-peroxidase-conjugated anti-mouse EnVision + secondary were purchased from Dako (Carpinteria, CA, IHC-Prediluted). Heatinactivated pooled human serum was purchased from Innovative Biologics (Herndon, VA). Dulbecco's Modified-Eagle's Media (DMEM) was purchased from Corning Life Sciences (Tewksbury, MA). Flash chromatography was performed using 32-63 $\mu \mathrm{m}$ flash silica gels from SiliCycle Inc. (Quebec, Canada). Reactions were monitored by thin-layered chromatography on precoated silica plates (250 $\mu \mathrm{m}, \mathrm{F}-254)$ from SiliCycle Inc. (Quebec, Canada). The compounds were visualized by UV fluorescence. CEM-ss CD4+ T cells were obtained from the HIV Reagent Program. 
Synthesis of L-phenylalanine and L-alanine docosyl esters. N-(carbobenzyloxy)- L- phenylalanine (2 $\mathrm{g}, 1$ equiv.), or $\mathrm{N}$-(carbobenzyloxy)- L- alanine (2 $\mathrm{g}, 1$ equiv.), and 1-docosanol (1.1 equiv.) were suspended in anhydrous DMF $(20 \mathrm{ml})$ and $\mathrm{CHCl}_{3}(20 \mathrm{~mL})$ and cooled to $-10{ }^{\circ} \mathrm{C}$ on a regular ice/ $\mathrm{NaCl}$ bath. To this mixture, HATU (1.5 equiv), and imidazole ( 1 equiv) were then added. $\mathrm{Next}, \mathrm{Et}_{3} \mathrm{~N}$ (2 equiv) was added to the pre-cooled mixture $\left(-10^{\circ} \mathrm{C}\right)$ dropwise. The resultant solution was stirred and gradually warmed to $45^{\circ} \mathrm{C}$ over $24 \mathrm{~h}$ under an inert argon atmosphere. The reaction mixture was concentrated on a rotary evaporator to yield a light-yellow solid that was purified by silica column flash chromatography eluting with a 4:1 mixture of hexanes and ethyl acetate. Next, the Cbz-protected amino acid esters were dissolved in $30 \mathrm{ml} \mathrm{CHCl}_{3}$ and $30 \mathrm{ml} \mathrm{MeOH}$, and deprotected using palladium on carbon $\left(\mathrm{Pd} / \mathrm{C}, 40 \%\right.$ weight of $\mathrm{N}-\mathrm{Cbz}$ - amino acid) and $\mathrm{Et}_{3} \mathrm{Si}(15$ equiv). After $24 \mathrm{~h}$, the reaction was filtered through celite, concentrated, and precipitated from diethyl ether to yield amorphous white powders of the desired free amino acid ester compounds that were used directly in the subsequent steps.

Synthesis and characterization of TFV ProTides. Monophenyl tenofovir ( $2 \mathrm{~g}$, $5.5 \mathrm{mmol}, 1$ equiv) was dried from anhydrous benzene $(15 \mathrm{~mL})$, resuspended in anhydrous ACN $(25 \mathrm{~mL})$, and then cooled at $-10^{\circ} \mathrm{C}$ under an argon atmosphere. After the addition of 5 equivalents of $\mathrm{SOCl}_{2}$ the reaction was heated at $65^{\circ} \mathrm{C}$ for $2 \mathrm{~h}$ under protection from light. The mixture was then dried from $15 \mathrm{ml}$ benzene by rotary evaporation. The chlorinated compound was then resuspended in $15 \mathrm{ml}$ of ACN and $15 \mathrm{ml} \mathrm{THF}$, and cooled to $-10^{\circ} \mathrm{C}$. A solution of L-phenylalanine docosyl ester $(2 \mathrm{~g}, 4.22 \mathrm{mmol}, 1$ equiv), or L-alanine docosyl ester $(2 \mathrm{~g}, 5.06 \mathrm{mmol}, 1$ equiv) was then added followed by dropwise addition of $\mathrm{Et}_{3} \mathrm{~N}$ (4 equiv) at $-10^{\circ} \mathrm{C}$. The reaction mixture was then warmed to room temperature, then heated at $45^{\circ} \mathrm{C}$ for $24 \mathrm{~h}$ under protection from light. The mixture was then concentrated to remove solvents. The residue was purified by silica column flash chromatography, eluting with $95 \%$ then $92.5 \%$ DCM in methanol. The desired compound fractions from the columns were dried on a rotary evaporator and freeze-dried from a mixture of diethyl ether/ water to obtain white powders, with chemical yields of 50-65\%. FTIR analysis was performed on a Spectrum Two FT-IR spectrometer (PerkinElmer, Waltham, MA, USA).

MALDI-TOF analysis. Samples were re-suspended in TFA (30\% Acetonitrile and $0.1 \%$ Trifluoroacetic Acid) and diluted to $\leq 1 \mathrm{ug} / \mathrm{ul}$. The MALDI matrix 2,5Dihydroxybenzoic Acid (DHB) was re-suspended in TA30 with a working concentration of $40 \mathrm{ug} / \mathrm{ul}$ and combined with the sample in a 1:1 ratio that was spotted on a Bruker MTP 384 ground steel target plate and allowed to dry. All spectra were recorded using a Bruker Autoflex Max MALDI-TOF mass spectrometer equipped with a smartbeam-II solid-state laser operating in positive reflectron mode and controlled with Bruker's flexControl (V3.4) instrument software. For each sample, spectra were acquired within a mass range of $500-3,500 \mathrm{Da}$ at an acquisition rate of 2,000 Hz. The remaining instrument parameters were configured as follows: ion source $1=19.00 \mathrm{kV}$, ion source $2=16.75 \mathrm{kV}$, lens $=8.50 \mathrm{kV}$, reflector $=21.00 \mathrm{kV}$, reflector $2=9.60 \mathrm{kV}$, pulsed ion extraction time $=130 \mathrm{~ns}$, and matrix suppression $\leq 450 \mathrm{Da}$. All raw spectra were processed with Bruker's flexAnalysis (V3.4) to include automated baseline subtraction and smoothing.

\section{Ultraperformance liquid chromatography-ultraviolet/visible drug and pro- drug quantifications. A Waters ACQUITY ultraperformance liquid chromato-} graphy (UPLC) H-Class system with a UV detector and Empower 3 software was used to measure the drug concentrations. M1TFV and M2TFV samples were separated on a Phenomenex Kinetex $5 \mu \mathrm{m} \mathrm{C18} \mathrm{column}(150 \times 4.6 \mathrm{~mm})$. TAF samples were separated on a Phenomenex Kinetex $5 \mu \mathrm{m} \mathrm{C18} \mathrm{column}$ $(150 \times 4.6 \mathrm{~mm})$. TAF was detected at $260 \mathrm{~nm}$ using an isocratic elution with a mobile phase of $40 \% 7.5 \mathrm{mM}$ ammonium acetate $\mathrm{pH} 4 / 60 \% \mathrm{MeOH}$ with a flow rate of $0.25 \mathrm{ml} / \mathrm{min}$. M1TFV and M2TFV were detected at 210 and $254 \mathrm{~nm}$ using an isocratic elution with a mobile phase of $98 \% \mathrm{MeOH} / 2 \% 7.5 \mathrm{mM}$ ammonium bicarbonate $\mathrm{pH} 7.0$ at a flow rate of $0.2 \mathrm{ml}$ min. The drug content was determined relative to the peak areas of drug standards $(0.05-50 \mu \mathrm{g} / \mathrm{ml})$ in $\mathrm{MeOH}$.

Photodiode-array detection (PDA) assessment of purity. A Waters Acquity H-Class Plus UPLC with PDA detector was used to qualitatively assess compound purity. M1TFV/M2TFV Method: 98\% HPLC-grade Methanol, 2\% $7.5 \mathrm{mM}$ Ammonium Bicarbonate $\mathrm{pH}$ 7.0; flow $1.0 \mathrm{~mL} / \mathrm{min}$; column - Phenomenex Kinetex $5 \mu \mathrm{m} \mathrm{C18} 100 \mathrm{~A}, 150 \times 4.6 \mathrm{~mm}$ with Phenomenex SecurityGuard and C18 $4 \times 3.0 \mathrm{~mm}$ guard cartridge; 3D data with lambda $190-490 \mathrm{~nm}$, resolution $1.2 \mathrm{~nm}$, sampling rate 10 points/sec on a Waters Acquity H-Class Plus UPLC with PDA detector. TAF Method: 60\% HPLC-grade Methanol, $40 \% 7.5 \mathrm{mM}$ Ammonium Acetate $\mathrm{pH} 4.0$; flow $0.500 \mathrm{~mL} / \mathrm{min}$; column - Phenomenex Kinetex $5 \mu \mathrm{m} \mathrm{C} 18$ $100 \mathrm{~A}, 150 \times 4.6 \mathrm{~mm}$ with Phenomenex SecurityGuard and C18 $4 \times 3.0 \mathrm{~mm}$ guard cartridge; 3D data with lambda $190-490 \mathrm{~nm}$, resolution $1.2 \mathrm{~nm}$, sampling rate 10 points/s on a Waters Acquity H-Class Plus UPLC with PDA detector.

Solubility. The aqueous and 1-octanol solubility of TAF, M1TFV, and M2TFV were determined by adding an excess of drug to water or 1-octanol in amber glass vials at room temperature and placed on a Thermo Scientific 4625 Plate Shaker at $100 \mathrm{rpm}$ for $48 \mathrm{~h}$. Samples were centrifuged at 20,000 $\times g$ for $10 \mathrm{~min}$ to separate the solubilized drug from insoluble pellet. Aqueous supernatants were frozen, lyophilized, and then resuspended in $\mathrm{MeOH}$. 1-octanol supernatants were prepared for analysis by dilution in $\mathrm{MeOH}$ and samples analyzed for drug content by UPLCUV (Waters Acquity H Class).

Nanoparticle synthesis and characterization. NM1TFV, NM2TFV, and NTAF solid drug nanoparticles were manufactured by high-pressure homogenization in aqueous buffers stabilized by non-ionic surfactants. The prodrug to surfactant ratio was maintained at 2:1 (w/w), and the suspension concentration was in the range $0.1-10 \% \mathrm{w} / \mathrm{v}$ for the drug/prodrug and $0.05-5 \% \mathrm{w} / \mathrm{v}$ for P407. Both NM1TFV and NM2TFV nanoformulations were prepared in P407 surfactant solution in $10 \mathrm{mM}$ HEPES at pH 7. For NTAF, a mixture of P407 and PEG 3350 was used as stabilizers. Attempts to formulate TAF using P407 without PEG 3350 produced unstable formulations. Specifically, TAF was dispersed in a P407/PEG 3350 solution in $10 \mathrm{mM}$ HEPES $\mathrm{pH} 5.5$ and allowed to form a pre-suspension. The prodrug to P407 to PEG 3350 ratio was maintained at 4:1:1 (w/w), and the suspension concentration was in the range $0.1-10 \% \mathrm{w} / \mathrm{v}$ for the drug/prodrug and $0.025-2.5 \%$ w/v for P407/PEG. The pre-suspensions were homogenized on an Avestin EmulsiFlex-C3 high-pressure homogenizer at 15,000-20,000 psi until the desired particle size of $250-350 \mathrm{~nm}$ was achieved. The homogenized solid drug nanoparticles were then evaluated for particle size, homogeneity, and surface charge by dynamic light scattering using a Malvern Zetasizer Nano-ZS. Long-term nanoparticle stability for all the formulations during storage was evaluated at room temperature over a period of 3 months. The amount of TAF, M1TFV, and M2TFV within the formulations after homogenization was determined from diluted formulation samples in $\mathrm{MeOH}(1,000-10,000$-fold dilution) and analyzed by UPLC-ultraviolet/visible (UV/vis) spectroscopy using calibration curves with known standards. The percentage of drug entrapped within each nanosuspension was calculated using the equation: encapsulation efficiency $(\%)=$ (weight of the drug in formulation/initial weight of drug added) $\times 100$.

Prodrug chemical stability. The $\mathrm{pH}$ buffers included $0.1 \%$ formic acid ( $\mathrm{pH} 2.0$ ), $7.5 \mathrm{mM}$ ammonium acetate buffer ( $\mathrm{pH} 6.0$ adjusted with acetic acid), PBS (pH 7.0) $7.5 \mathrm{mM}$ ammonium bicarbonate buffer ( $\mathrm{pH} 8.0$ adjusted with acetic acid), and $0.1 \%$ ammonium hydroxide ( $\mathrm{pH} 10.3$ and stored at $4{ }^{\circ} \mathrm{C}$ until use. NM1TFV or NTAF were incubated at $37^{\circ} \mathrm{C}$ in $100 \mu \mathrm{L}$ of the buffer. The final concentration of the drug in the incubation mixture was $1 \mu \mathrm{M}$. The reactions were stopped by the addition of $900 \mu \mathrm{L}$ of acidified methanol $(0.1 \%$ formic acid $+2.5 \mathrm{mM}$ ammonium formate $)$ at 0 $5 \mathrm{~s}, 30 \mathrm{~min}, 1,2,6,12$, and $24 \mathrm{~h}$ time points. Control samples were incubated using the same method as in the absence of substrates, however, substrates were added after the addition of acidified methanol. The mixtures were centrifuged at $16,000 \times g$ for $10 \mathrm{~min}$. The supernatants were aspirated and stored at $-80^{\circ} \mathrm{C}$ until ultraperformance liquid chromatography tandem mass spectrometry (UPLC-MS/MS) analysis.

Human monocyte-derived macrophage (MDM) isolation, cultivation, and cytotoxicity. Human monocytes were obtained, cultured, and differentiated into MDM as previously described ${ }^{11,12,34,62}$. The cell viability after treatment with nanoparticles was evaluated using the MTT assay. Human MDMs plated in 96-well plates at a density of $0.08 \times 10^{6}$ cells/well were exposed to a range of concentrations $(1.5625-200 \mu \mathrm{M})$ of NM1TFV, NM2TFV, or TAF for $8 \mathrm{~h}$. The cells were then washed with PBS after which $100 \mu \mathrm{L}$ of MTT solution was added to each well $(5 \mathrm{mg} / \mathrm{mL})$ for $45 \mathrm{~min}$ at $37^{\circ} \mathrm{C}$. The MTT solution was then replaced with $200 \mu \mathrm{L} /$ well of DMSO. A Molecular Devices SpectraMax M3 plate reader with SoftMax Pro 6.2 software (Sunnyvale, CA) was used to record absorbance at a wavelength of $490 \mathrm{~nm}$.

Drug nanoformulation uptake and retention. Human MDMs were used for in vitro formulation assessments. MDM formulation uptake and retention studies were performed in flat-bottom, 12 -well plates at a density of $1.2 \times 10^{6}$ cells/well, with each treatment completed in triplicate. For cellular uptake studies, MDMs were treated with $10 \mu \mathrm{M}$ or $100 \mu \mathrm{M}$ NM1TFV, NM2TFV, and TAF. At 2,4 , and $8 \mathrm{~h}$ after treatment, the cells were collected, processed, and analyzed for drug content by UPLC-UV/vis as previously described $12,13,56,63$. For the retention studies, MDMs were treated with $10 \mu \mathrm{M}$ or $100 \mu \mathrm{M}$ NM1TFV, NM2TFV, or NTAF for $8 \mathrm{~h}$, and then washed twice with $1 \mathrm{ml}$ PBS. Then, a culture medium (without drug) was added, and a half-media volume was replaced every other day. MDMs were collected at days $1,5,10,15$, and 30 after treatment, and then analyzed for intracellular drug content by UPC-UV/vis. For TEM imaging of intracellular nanoparticles, MDMs were treated with $10 \mu \mathrm{M}$ NM1TFV, NM2TFV, or TAF for $8 \mathrm{~h}$ and then washed twice with PBS. MDMs were then collected immediately after the $8 \mathrm{~h}$ treatment and analyzed by an FEI Tecnai G2 Spirit transmission electron microscope as previously described ${ }^{11,12,14}$.

Antiretroviral efficacy. To determine long-term antiretroviral efficacy following a single treatment, MDMs were plated in flat-bottom 12-well plates at a density of $1.2 \times 10^{6}$ cells/well. MDMs were treated with $10 \mu \mathrm{M}$ or $100 \mu \mathrm{M}$ NM1TFV, NM2TFV, NTAF, or TAF for $8 \mathrm{~h}$. After treatment, the cells were washed twice with PBS and cultured in a fresh culture medium without drug with half-media 
replacement every other day. On days $1,5,10,15,20,25$, and 30 following treatment, the cells were infected with $\mathrm{HIV}-1_{\mathrm{ADA}}$ (a macrophage-tropic viral strain) at a multiplicity of infection of 0.1 infectious particles/cell for $8 \mathrm{~h}$. MDM were cultured, and media samples collected on day 10 for the measurement of HIV reverse transcriptase (RT) activity as previously described ${ }^{64,65}$. Cells were fixed in $2 \%$ PFA at each time point, and expression of the HIV-1p24 antigen was determined by immunocytochemistry. To determine the $\mathrm{IC}_{50}$ of each prodrug and its nanoformulation, MDMs were plated in flat-bottom 96 -well plates $\left(0.08 \times 10^{6}\right.$ cells/ well). Cells were treated with a range of drug concentrations, $0.00005-5,000 \mathrm{nM}$, of TAF, M1TFV, M2TFV, NTAF, NM1TFV, or NM2TFV for $1 \mathrm{~h}$ prior to infection with HIV-1 $1_{\mathrm{ADA}}$ (multiplicity of infection of 0.1 ) for $4 \mathrm{~h}$. After $4 \mathrm{~h}$ of viral challenge, the cells were washed with PBS and given fresh media that contained the same concentrations of drug $(0.00005-5,000 \mathrm{nM})$. Cell supernatants were collected 10 days later and assayed for HIV-1 RT activity as described above. Replicate studies were performed in CEM-ss CD4+ T-cell cultures. Specifically, cells were plated in 96-well plates, centrifuged at $650 \times g$ for $5 \mathrm{~min}$, and resuspended in (0.1-1,000 nM) drug-containing media for $1 \mathrm{~h}$. The subsequent challenge with

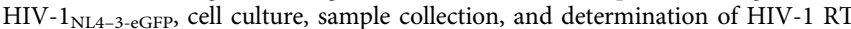
activity were performed as previously reported ${ }^{13,15}$.

PK studies. Twelve-week-old healthy Sprague Dawley rats (male, 300-350 g; female, 200-230 g; SASCO) were purchased from Charles River Laboratories (Wilmington, MA) and housed in the University of Nebraska Medical Center (UNMC) laboratory animal facility according to the American Animal Association and Laboratory Animal Care guidance. All procedures were approved by the Institutional Animal Care and Use Committee at the University of Nebraska Medical Center (UNMC) as set forth by the National Institutes of Health (NIH). Following a one-week quarantine, the animals were randomized by weight and administered a single intramuscular injection (IM, caudal thigh muscle; $200 \mu \mathrm{L}$ ) of $75 \mathrm{mg} / \mathrm{kg}$ TFV equivalents of either NM1TFV, NM2TFV, or NTAF (males) or NM2TFV or NTAF (females). On days 1, 14, 28, 42, and 56 after injection, blood samples were collected from non-fasted animals into EDTA tubes via the retroorbital sinus using autoclaved glass capillaries. A $25 \mu \mathrm{L}$ aliquot of blood was immediately diluted into $1 \mathrm{~mL}$ of $\mathrm{MeOH}$ (containing $2.5 \mathrm{mM}$ ammonium formate and $0.1 \%$ formic acid) for drug quantitation. The remaining $500 \mu \mathrm{L}-1 \mathrm{~mL}$ of whole blood was used for PBMC isolation. On day 28 (females) or days 7, 28, and 56 (males) post-drug administration, non-fasted animals were humanely euthanized. Beginning in the morning, all animals treated with NM1TFV were first terminated, followed by all NM2TFV, then all NTAF. Spleen, lymph node, adipose, testes, liver, lung, gut (duodenum/jejunum), kidney, brain, vaginal and rectal tissue were collected for the quantitation of TFV and prodrugs. Additionally, rat PBMCs, splenocytes, lymphocytes, and liver parenchymal and non-parenchymal cells were isolated on sacrifice days for analysis of TFV-DP content. The amount of TFV, M1TFV, and M2TFV in blood and tissues were quantified from calibration curves with known standards by UPLC-MS/MS using a Waters ACQUITY H-class UPLC connected to a Xevo TQ-S micro mass spectrometer. The solvents used for sample processing and analytical methods were MS-grade (Fisher). Serum chemistry profile, animal body weights, organ to body weight ratios, complete blood counts (CBCs), and histological evaluations were used to assess adverse formulation reactions. Histological examination was performed on $5 \mu \mathrm{m}$ tissue sections stained with hematoxylin and eosin staining. The images were captured on a Nuance EX multispectral imaging system affixed to a Nikon Eclipse E800 microscope (Nikon Instruments) and evaluated by a certified pathologist in accordance with the guidelines of the Society of Toxicologic Pathology. Serum chemistry data sets were acquired on a VetScan comprehensive diagnostic profile disc and a VetScan VS-2 instrument (Abaxis Veterinary Diagnostics). Biochemical analyses of CBCs were performed using an Abaxis Vetscan HM5 Hematology Analyzer. For both, the results for treated animals were compared with those from age- and sex-matched untreated control rats.

TFV-DP sample preparation. Intracellular TFV-DP was extracted from human MDM or rat PBMCs as follows. MDM was treated with $10 \mu \mathrm{M}$ or $100 \mu \mathrm{M}$ NM1TFV, NM2TFV, or TAF. At 2, 4, and $8 \mathrm{~h}$ after treatment, MDM was washed with PBS to remove the excess free drug. The cells were then collected in 70\% MSgrade $\mathrm{MeOH}$. TFV-DP from MDM was extracted as follows. Sep-Pak QMA cartridges (360 mg, 37-55 $\mu \mathrm{m}$; Waters, Milford, MA) were used to separate TFV-DP from TFV- and mono-phosphate counterparts. The QMA cartridges were conditioned with $10 \mathrm{ml}$ of $500 \mathrm{mM} \mathrm{KCl}$, followed by $10 \mathrm{ml}$ of $5 \mathrm{mM} \mathrm{KCl}$. The sample $(200 \mu \mathrm{L})$ was loaded onto the cartridges and washed with $12 \mathrm{ml}$ of $75 \mathrm{mM} \mathrm{KCl}$. The triphosphate (TP) fraction was eluted with $3 \mathrm{ml}$ of $500 \mathrm{mM} \mathrm{KCl}$. The $\mathrm{pH}$ of the TP fraction was lowered to 4.25 by adding $45 \mu \mathrm{l}$ ammonium acetate buffer ( $\mathrm{pH} 4$, $10 \mathrm{mM}$ ) per ml eluate, and dephosphorylated by adding one unit of type XA sweet potato acid phosphatase (Sigma-Aldrich) per ml eluate ( $3 \mu \mathrm{L}$ per sample) and incubating at $37^{\circ} \mathrm{C}$ for $45 \mathrm{~min} .150 \mu \mathrm{L}$ of $12 \%$ TFA and $10 \mu \mathrm{L}$ of $5 \mathrm{ng} / \mathrm{mL}^{15} \mathrm{~N}_{2}$ ${ }^{13} \mathrm{C}-3 \mathrm{TC}$ and $\mathrm{d} 4-\mathrm{ABC}$ internal standard were then added. Samples were then loaded onto Waters OASIS HLB cartridges (60 mg, $30 \mu \mathrm{m}$; Waters, Milford, MA) pre-conditioned with $3 \mathrm{ml} \mathrm{100 \%}$ methanol followed by $3 \mathrm{ml} 1 \%$ TFA to remove salts. Salts were removed from the dephosphorylated samples with $3.5 \mathrm{ml}$ of $1 \%$ TFA in water, then eluted with $1.5 \mathrm{ml} 100 \%$ methanol and evaporated under vacuum. Once dry, the residue was stored at $-80^{\circ} \mathrm{C}$ until UPLC-MS/MS analyses.
Samples were reconstituted in $100 \mu \mathrm{L}$ of $10 \% \mathrm{MeOH}, 90 \% 7.5 \mathrm{mM}$ ammonium bicarbonate $\mathrm{pH} 7$ in LC-MS grade water.

PBMC isolation from whole blood. PBMCs were isolated from $\sim 500 \mu \mathrm{L}$ to $1 \mathrm{~mL}$ volumes of whole blood collected by retro-orbital sinus punctures then placed into EDTA-coated tubes. The whole blood was layered on top of an equal volume of Histoplaque $^{\circledR}-1083$ (Sigma-Aldrich) at room temperature and centrifuged at $400 \times g$ for $30 \mathrm{~min}$ at room temperature with an off-brake setting. The PBMCs were then collected from the plasma/Histoplaque interface into a $5 \mathrm{~mL}$ conical centrifuge tube containing $3 \mathrm{~mL}$ sterile PBS and centrifuged at $250 \times g$ for $10 \mathrm{~min}$ at room temperature. Following aspiration of the supernatant, the pellet was resuspended in $1.5 \mathrm{~mL}$ of sterile PBS and centrifuged at $250 \times g$ for $10 \mathrm{~min}$. The procedure was repeated twice. Following cell counting, the PBMCs were lysed in $200 \mu \mathrm{L}$ of LC-MS grade $70 \% \mathrm{MeOH}, 30 \%$ water, and stored at $-80{ }^{\circ} \mathrm{C}$.

TFV-DP extraction from mucosal tissues. On day 28 (female rats) or days 28 and 56 (male rats) post drug treatment, rats were humanely euthanized. Rectal and vaginal tissues were collected, flash-frozen in liquid nitrogen, and stored at $-80^{\circ} \mathrm{C}$ until further processing. For TFV-DP quantitation, vaginal and rectal tissues were homogenized in $50 \%$ and $70 \% \mathrm{MeOH}$ in water, respectively, using a Qiagen TissueLyser II. TFV-DP was extracted from the homogenates. Individual cell populations were not isolated from the tissues. TFV-DP samples were further processed and analyzed as described herein.

Sample preparation and UPLC-MS/MS analyses. TFV, M1TFV, and M2TFV in biological samples were quantitated by UPLC-MS/MS using a Waters Acquity $\mathrm{H}$-class UPLC connected to a Waters Xevo TQ-S micro mass spectrometer (Milford, MA). For blood and tissue analysis of TFV, $10 \mu \mathrm{L}$ of internal standard (IS) solution $(50 \mathrm{ng} / \mathrm{ml} 3 \mathrm{TC}-\mathrm{d} 3)$ was added to each sample. Lymph node and spleen samples were homogenized in nine volumes of $90 \% \mathrm{MeOH}(0.1 \%$ Formic acid + $2.5 \mathrm{mM}$ ammonium formate), while all other tissues were homogenized in four volumes of $90 \% \mathrm{MeOH}$ ( $0.1 \%$ Formic acid $+2.5 \mathrm{mM}$ ammonium formate). $1 \mathrm{~mL}$ $\mathrm{MeOH}(0.1 \%$ Formic acid $+2.5 \mathrm{mM}$ ammonium formate) was added to $25 \mu \mathrm{L}$ of whole blood to extract TFV. Samples were separated on a CSH C18 $(1.7 \mu \mathrm{m}, 2.1 \times$ $100 \mathrm{~mm}$ ) column using a mobile phase consisting of $7.5 \mathrm{mM}$ ammonium bicarbonate adjusted to $\mathrm{pH} 7$ (mobile phase A) and 100\% Optima MS-grade methanol (mobile phase B) at a flow rate of $0.22 \mathrm{~mL} / \mathrm{min}$ and run time of $8 \mathrm{~min}$. TFV was detected in the positive ionization mode using multiple reaction monitoring (MRM) transitions for TFV and 3TC-d3, of $288.23>159.01$, and

$233.23>114.97 \mathrm{~m} / \mathrm{z}$, respectively. The initial mobile phase composition was $5 \% \mathrm{~B}$ for the first $4.25 \mathrm{~min}$ at which time it was increased to $30 \% \mathrm{~B}$ over $0.25 \mathrm{~min}$, then increased to $90 \% \mathrm{~B}$ and held for a further $0.6 \mathrm{~min}$ before returning to initial conditions. Spectra were analyzed and quantified by MassLynx software version 4.1. Quantitation was based upon drug peak area to internal standard peak area ratios.

For quantitation of M1TFV and M2TFV in blood and tissues, $10 \mu \mathrm{L}$ of internal standard (IS) solution ( $500 \mathrm{ng} / \mathrm{ml}$ of stearoylated darunavir (SDRV)) was added to each sample. Lymph node and spleen samples were homogenized in nine volumes of $90 \% \mathrm{MeOH}(0.1 \%$ Formic acid $+2.5 \mathrm{mM}$ ammonium formate), while all other tissues were homogenized in four volumes of $90 \% \mathrm{MeOH}(0.1 \%$ Formic acid + $2.5 \mathrm{mM}$ ammonium formate). $1 \mathrm{~mL} \mathrm{MeOH}(0.1 \%$ Formic acid $+2.5 \mathrm{mM}$ ammonium formate) was added to $25 \mu \mathrm{L}$ of whole blood to extract prodrug. Chromatographic separation of $10 \mu \mathrm{L}$ sample injections of M1TFV/ M2TFV was achieved with an ACQUITY UPLC-BEH Shield RP18 column $(1.7 \mu \mathrm{m}, 2.1 \times$ $30 \mathrm{~mm})$ using a gradient of mobile phase A $(7.5 \mathrm{mM}$ ammonium formate in Optima-grade water adjusted to $\mathrm{pH} 3$ using formic acid) and mobile phase $\mathrm{B}$ (100\% Optima-grade methanol) at a flow rate of $0.35 \mathrm{~mL} / \mathrm{min}$. The initial mobile phase composition was $88 \%$ B for the first 5 min at which time it was increased to $95 \%$ B over 0.25 mins and held constant for $1.50 \mathrm{~min}$. Prodrugs were detected in the positive ionization mode using MRM transitions for M1TFV, M2TFV, and SDRV, of $819.70>206.10,743.68>206.10$, and $814.70>155.96 \mathrm{~m} / \mathrm{z}$, respectively. Spectra were analyzed and quantified by MassLynx software version 4.1. Quantitation was based upon drug peak area to internal standard peak area ratios.

TFV-DP: Parent TFV was generated from TFV-DP during sample preparation and then subjected to UPLC-MS/MS analyses. The UPLC-MS/MS system was comprised of a Waters ACQUITY UPLC system coupled to a Waters Xevo TQ-XS triple quadrupole mass spectrometer (Waters, Milford, MA) with electrospray ionization (ESI) source. A CSH C18 analytical column $(2.1 \times 100 \mathrm{~mm}, 1.7 \mu \mathrm{m})$ equipped with a guard column (Waters, Milford, MA) at $30^{\circ} \mathrm{C}$ was used for analyte separation. Samples were maintained at $4{ }^{\circ} \mathrm{C}$ during analysis. The MRM transitions of $288.10<159.04 \mathrm{~m} / \mathrm{z}$ and $232.97<115.00 \mathrm{~m} / \mathrm{z}$ were used for TFV and 3TC-d3 internal standard (IS) quantification, respectively. Mobile phase A consisted of $7.5 \mathrm{mM}$ ammonium bicarbonate in water (MS grade, Fisher) with $\mathrm{pH}$ adjusted to 7.0 with glacial acetic acid (ACS grade, Sigma). Mobile phase B was $100 \%$ methanol (MS grade, Fisher). The flow rate was $0.22 \mathrm{~mL} / \mathrm{min}$. A gradient of $95 \% \mathrm{~A}$ and $5 \% \mathrm{~B}$ was held for $4.25 \mathrm{~min}$, and then B was increased to $30 \%$ over $0.25 \mathrm{~min}$. B was then held at $90 \%$ for $0.4 \mathrm{~min}$ before returning to initial conditions. Spectra were analyzed and quantified by MassLynx software version 4.1. Quantitation was based upon drug peak area to internal standard peak area ratios. 
Statistical analyses. Statistical analyses of data sets were performed on a GraphPad Prism v9.0.0.0 software and Microsoft Excel. Both in vitro and in vivo study data sets are expressed as means \pm SEM with a minimum of three biological replicates for each study. The Student's t-test (two-tailed, paired, and unpaired) was used to compare means between two groups while a one-way ANOVA followed by Tukey's post hoc test was used for comparisons between three or more groups. Statistical significances were denoted as ${ }^{*} P<0.05$, $* * P<0.01$, $* * * P<0.001$, and $* * * * P<0.0001$. Comparison between intramuscular and tissue drug concentrations was performed by Pearson correlation and linear regression models.

Study approvals. All animal studies were approved by the University of Nebraska Medical Center Institutional Animal Care and Use Committee in accordance with the standards incorporated in the Guide for the Care and Use of Laboratory Animals $^{66}$. Human monocytes were isolated by leukapheresis from HIV-1/2 and hepatitis B seronegative donors according to an approved University of Nebraska Medical Center Institutional Review Board exempt protocol. All donors gave informed consent for the use of the deidentified material.

Reporting summary. Further information on research design is available in the Nature Research Reporting Summary linked to this article.

\section{Data availability}

The data supporting the study's findings are available within the article and its supplementary files. All the relevant data used to generate main and supplementary figures are included as Source Data (https://doi.org/10.6084/m9.figshare.15168909). Source data are provided with this paper.

Received: 3 May 2021; Accepted: 20 August 2021;

Published online: 16 September 2021

\section{References}

1. Fauci, A. S., Redfield, R. R., Sigounas, G., Weahkee, M. D. \& Giroir, B. P. Ending the HIV epidemic: a plan for the United States. JAMA 321, 844-845 (2019).

2. Winner, B. et al. Effectiveness of long-acting reversible contraception. N. Engl. J. Med. 366, 1998-2007 (2012)

3. Adams, C. E., Fenton, M. K., Quraishi, S. \& David, A. S. Systematic metareview of depot antipsychotic drugs for people with schizophrenia. Br. J. Psychiatry 179, 290-299 (2001)

4. Gulick, R. M. \& Flexner, C. Long-acting HIV drugs for treatment and prevention. Annu Rev. Med. 70, 137-150 (2019).

5. Spreen, W. et al. GSK1265744 pharmacokinetics in plasma and tissue after single-dose long-acting injectable administration in healthy subjects. J. Acquir Immune Defic. Syndr. 67, 481-486 (2014).

6. CABENUVA. Offers adults living with HIV a new once-monthly injectable option for maintaining viral suppression. https://www.janssen.com/janssenannounces-us-fda-approval-cabenuva-rilpivirine-and-cabotegravir-first-longacting-regimen (2021).

7. ViiV Healthcare. Presents positive 48-week data from phase III study showing every-two-month regimen of investigational long-acting, injectable cabotegravir and rilpivirine has similar efficacy to once-monthly dosing. https://viivhealthcare.com/en-gb/media/press-releases/2020/march/viivhealthcare-presents-positive-48-week-data-from-phase-iii-s/ (2020).

8. Swindells, S. et al. Long-acting cabotegravir and rilpivirine for maintenance of HIV-1 suppression. N. Engl. J. Med 382, 1112-1123 (2020).

9. Markowitz, M. et al. Safety and tolerability of long-acting cabotegravir injections in HIV-uninfected men (ECLAIR): a multicentre, double-blind, randomised, placebo-controlled, phase 2a trial. Lancet HIV 4, e331-e340 (2017).

10. Margolis, D. A. et al. Long-acting intramuscular cabotegravir and rilpivirine in adults with HIV-1 infection (LATTE-2): 96-week results of a randomised, open-label, phase 2b, non-inferiority trial. Lancet 390, 1499-1510 (2017).

11. Kulkarni, T. A. et al. A year-long extended release nanoformulated cabotegravir prodrug. Nat. Mater. 19, 910-920 (2020).

12. Sillman, B. et al. Creation of a long-acting nanoformulated dolutegravir. Nat. Commun. 9, 443 (2018).

13. Hilaire, J. R. et al. Creation of a long-acting rilpivirine prodrug nanoformulation. J. Control Release 311-312, 201-211 (2019).

14. Banoub, M. G. et al. Synthesis and characterization of long-acting Darunavir prodrugs. Mol. Pharm. 17, 155-166 (2020)

15. Smith, N. et al. A long acting nanoformulated lamivudine ProTide. Biomaterials 223, 119476 (2019).

16. Edagwa, B., McMillan, J., Sillman, B. \& Gendelman, H. E. Long-acting slow effective release antiretroviral therapy. Expert Opin. Drug Deliv. 14, 1281-1291 (2017).
17. McGuigan, C., Pathirana, R. N., Mahmood, N., Devine, K. G. \& Hay, A. J. Aryl phosphate derivatives of AZT retain activity against HIV1 in cell lines which are resistant to the action of AZT. Antivir. Res. 17, 311-321 (1992).

18. De Clercq, E. Tenofovir alafenamide (TAF) as the successor of tenofovir disoproxil fumarate (TDF). Biochem. Pharm. 119, 1-7 (2016).

19. Gibson, A. K., Shah, B. M., Nambiar, P. H. \& Schafer, J. J. Tenofovir Alafenamide. Ann. Pharmacother. 50, 942-952 (2016).

20. Golla, V. M., Kurmi, M., Shaik, K. \& Singh, S. Stability behaviour of antiretroviral drugs and their combinations. 4: Characterization of degradation products of tenofovir alafenamide fumarate and comparison of its degradation and stability behaviour with tenofovir disoproxil fumarate. J. Pharm. Biomed. Anal. 131, 146-155 (2016).

21. Gunawardana, M. et al. Pharmacokinetics of long-acting tenofovir alafenamide (GS-7340) subdermal implant for HIV prophylaxis. Antimicrob. Agents Chemother. 59, 3913-3919 (2015).

22. Sizovs, A. et al. Trans-urocanic acid enhances tenofovir alafenamide stability for long-acting HIV applications. Int. J. Pharm. 587, 119623 (2020).

23. Prathipati, P. K., Mandal, S., Pon, G., Vivekanandan, R. \& Destache, C. J. Pharmacokinetic and tissue distribution profile of long acting tenofovir alafenamide and elvitegravir loaded nanoparticles in humanized mice model. Pharm. Res. 34, 2749-2755 (2017).

24. Hobson, J. J. et al. Branched copolymer-stabilised nanoemulsions as new candidate oral drug delivery systems. RSC Adv. (in the press) (2018).

25. Mandal, S. et al. Nanoencapsulation introduces long-acting phenomenon to tenofovir alafenamide and emtricitabine drug combination: a comparative pre-exposure prophylaxis efficacy study against HIV-1 vaginal transmission. $J$. Control Release 294, 216-225 (2019).

26. Katz, D. H. et al. n-docosanol: broad spectrum anti-viral activity against lipidenveloped viruses. Ann. N. Y Acad. Sci. 724, 472-488 (1994).

27. Marcelletti, J. F. Synergistic inhibition of herpesvirus replication by docosanol and antiviral nucleoside analogs. Antivir. Res. 56, 153-166 (2002).

28. McGuigan, C. et al. Application of phosphoramidate pronucleotide technology to abacavir leads to a significant enhancement of antiviral potency. J. Med. Chem. 48, 3504-3515 (2005).

29. Anderson, P. L. et al. Emtricitabine-tenofovir concentrations and preexposure prophylaxis efficacy in men who have sex with men. Sci. Transl. Med. 4, 151ra125 (2012).

30. McGuigan, C. et al. Synthesis, anti-human immunodeficiency virus activity and esterase lability of some novel carboxylic ester-modified phosphoramidate derivatives of stavudine (d4T). Antivir. Chem. Chemother. 9, 473-479 (1998).

31. McGuigan, C., Tsang, H. W., Sutton, P. W., De Clercq, E. \& Balzarini, J. Synthesis and anti-HIV activity of some novel chain-extended phosphoramidate derivatives of d4T (stavudine): esterase hydrolysis as a rapid predictive test for antiviral potency. Antivir. Chem. Chemother. 9, 109-115 (1998).

32. Kandil, S. et al. ProTides of BVdU as potential anticancer agents upon efficient intracellular delivery of their activated metabolites. Bioorg. Med. Chem. Lett. 26, 5618-5623 (2016)

33. Bressani, R. F. et al. Pharmacotoxicology of monocyte-macrophage nanoformulated antiretroviral drug uptake and carriage. Nanotoxicology 5 592-605 (2011).

34. Nowacek, A. S. et al. NanoART synthesis, characterization, uptake, release, and toxicology for human monocyte-macrophage drug delivery. Nanomedicine 4, 903-917 (2009).

35. Guo, D. et al. Endosomal trafficking of nanoformulated antiretroviral therapy facilitates drug particle carriage and HIV clearance. J. Virol. 88, 9504-9513 (2014).

36. Panel on Antiretroviral Guidelines for Adults and Adolescents. Guidelines for the use of antiretroviral agents in adults and adolescents living with HIV. https://aidsinfo.nih.gov/ContentFiles/AdultandAdolescentGL.pdf (2020).

37. Terrault, N. A. et al. Update on prevention, diagnosis, and treatment of chronic hepatitis B: AASLD 2018 hepatitis B guidance. Clin. Liver Dis. 12, 33-34 (2018).

38. Cohen, M. S. Successful treatment of HIV eliminates sexual transmission. Lancet 393, 2366-2367 (2019).

39. Fonner, V. A. et al. Effectiveness and safety of oral HIV preexposure prophylaxis for all populations. AIDS 30, 1973-1983 (2016).

40. Eggleton, J. S. \& Nagalli, S. Highly Active Antiretroviral Therapy (HAART) (StatPearls, 2020)

41. Chan, L., Asriel, B., Eaton, E. F. \& Wyatt, C. M. Potential kidney toxicity from the antiviral drug tenofovir: new indications, new formulations, and a new prodrug. Curr. Opin. Nephrol. Hypertens. 27, 102-112 (2018).

42. Patterson, K. B. et al. Penetration of tenofovir and emtricitabine in mucosal tissues: implications for prevention of HIV-1 transmission. Sci. Transl. Med. 3, 112re114 (2011)

43. Cottrell, M. L. et al. Single-dose pharmacokinetics of tenofovir alafenamide and its active metabolite in the mucosal tissues. J. Antimicrob. Chemother. 72, 1731-1740 (2017). 
44. Chua, C. Y. X. et al. Transcutaneously refillable nanofluidic implant achieves sustained level of tenofovir diphosphate for HIV pre-exposure prophylaxis. $J$. Control Release 286, 315-325 (2018).

45. Johnson, L. M. et al. Characterization of a reservoir-style implant for sustained release of tenofovir alafenamide (TAF) for HIV pre-exposure prophylaxis (PrEP). Pharmaceutics https://doi.org/10.3390/pharmaceutics11070315 (2019).

46. Schlesinger, E. et al. A tunable, biodegradable, thin-film polymer device as a long-acting implant delivering tenofovir alafenamide fumarate for HIV preexposure prophylaxis. Pharm. Res. 33, 1649-1656 (2016).

47. Alexaki, A., Liu, Y. \& Wigdahl, B. Cellular reservoirs of HIV-1 and their role in viral persistence. Curr. HIV Res. 6, 388-400 (2008).

48. Darville, N. et al. Intramuscular administration of paliperidone palmitate extended-release injectable microsuspension induces a subclinical inflammatory reaction modulating the pharmacokinetics in rats. J. Pharm. Sci. 103, 2072-2087 (2014).

49. Darville, N. et al. Modeling the time course of the tissue responses to intramuscular long-acting paliperidone palmitate nano-/microcrystals and polystyrene microspheres in the rat. Toxicol. Pathol. 44, 189-210 (2016).

50. Darville, N. et al. The effect of macrophage and angiogenesis inhibition on the drug release and absorption from an intramuscular sustained-release paliperidone palmitate suspension. J. Control Release 230, 95-108 (2016).

51. van 't Klooster, G. et al. Pharmacokinetics and disposition of rilpivirine (TMC278) nanosuspension as a long-acting injectable antiretroviral formulation. Antimicrob. Agents Chemother. 54, 2042-2050 (2010).

52. Jucker, B. M. et al. Multimodal imaging approach to examine biodistribution kinetics of Cabotegravir (GSK1265744) long acting parenteral formulation in rat. J. Control Release 268, 102-112 (2017).

53. Keller, M. J. et al. Tenofovir disoproxil fumarate intravaginal ring for HIV preexposure prophylaxis in sexually active women: a phase 1 , single-blind, randomised, controlled trial. Lancet HIV 6, e498-e508 (2019).

54. Rodriguez-Garcia, M. et al. Tenofovir inhibits wound healing of epithelial cells and fibroblasts from the upper and lower human female reproductive tract. Sci. Rep. 8, 45725 (2017).

55. Weber, K. et al. Pathology in continuous infusion studies in rodents and nonrodents and ITO (Infusion Technology Organisation)-recommended protocol for tissue sampling and terminology for procedure-related lesions. J. Toxicol. Pathol. 24, 113-124 (2011).

56. Zhou, T. et al. Creation of a nanoformulated cabotegravir prodrug with improved antiretroviral profiles. Biomaterials 151, 53-65 (2018).

57. Su, J. T. et al. A subcutaneous implant of tenofovir alafenamide fumarate causes local inflammation and tissue necrosis in rabbits and macaques. Antimicrob. Agents Chemother. https://doi.org/10.1128/AAC.01893-19 (2020).

58. Denise A. et al. Long-acting tenofovir and nitazoxanide formulations suppress HBV replication. Abstract No. 610. Presented at: Conference on Retroviruses and Opportunistic Infections (CROI 2020); March 8-11 (Boston, MA, 2020).

59. Simpson, S. M. et al. Design of a drug-eluting subcutaneous implant of the antiretroviral tenofovir alafenamide fumarate. Pharm. Res. 37, 83 (2020).

60. ViiV Healthcare. Announces investigational injectable cabotegravir is superior to oral standard of care for HIV prevention in women. https:// viivhealthcare.com/en-us/us-news/us-articles/2020/viiv-healthcareannounces-investigational-injectable-cabotegravir-is-superior-to-oralstandard-of-care-for-hiv-prevention-in-women/ (2020).

61. Bollinger, R. C. et al. Addressing the global burden of hepatitis B virus while developing long-acting injectables for the prevention and treatment of HIV. Lancet HIV 7, e443-e448 (2020).

62. Gendelman, H. E. et al. Efficient isolation and propagation of human immunodeficiency virus on recombinant colony-stimulating factor 1-treated monocytes. J. Exp. Med. 167, 1428-1441 (1988).

63. Lin, Z. et al. ProTide generated long-acting abacavir nanoformulations. Chem. Commun. 54, 8371-8374 (2018).

64. Balkundi, S. et al. Comparative manufacture and cell-based delivery of antiretroviral nanoformulations. Int J. Nanomed. 6, 3393-3404 (2011).

65. Nowacek, A. S. et al. Nanoformulated antiretroviral drug combinations extend drug release and antiretroviral responses in HIV-1-infected macrophages: implications for neuroAIDS therapeutics. J. Neuroimmune Pharm. 5, 592-601 (2010).

66. Committee for the Update of the Guide for the Care and Use of Laboratory Animals. Guide for the Care and Use of Laboratory Animals. National Academies Press (2011).

\section{Acknowledgements}

We thank the University of Nebraska Medical Center (UNMC) cores for NMR (Ed Ezell), Elutriation and Cell Separation (Myhanh Che, $\mathrm{Na} \mathrm{Ly,} \mathrm{and} \mathrm{Li} \mathrm{Wu),} \mathrm{Electron} \mathrm{Microscopy}$ (Nicholas Conoan and Tom Bargar), Histology (Tissue Sciences Facility), MALDI/TOF Mass Spectrometry (Eric Troudt), compound purity by UPLC photodiode array detector (Brady Sillman), as well as Comparative Medicine for technical assistance and animal care. We also wish to thank Gilead Sciences Inc. for the supply of TAF. This research is supported by the University of Nebraska Foundation, which includes donations from the Carol Swarts, M.D. Emerging Neuroscience Research Laboratory, the Margaret R. Larson Professorship, the Frances and Louie Blumkin Endowment, and the Harriet Singer Endowment; the Vice Chancellor's Office of the University of Nebraska Medical Center for Core Facilities; Nickolus Badami Fellowship (to B.E.) and the National Institutes of Health grants 1R01AI145542-01A1, 1R56 AI138613-01A1, P01 DA028555, and P30 MH062261.

\section{Author contributions}

D.C.- -study design, synthesized the prodrugs and formulations, design and execution of most experiments, data acquisition, data analysis, and interpretation, co-wrote paper; N.S.- data acquisition and interpretation; S.D.- data acquisition and interpretation; A.B. - assisted with animal study design and data acquisition; B.L.S.D.--performed LCMS/MS analyses; J.M.--designed, supervised, and analyzed the mass spectrometry data; N.G.- development of LC-MS/MS methods, data acquisition and interpretation; Y.A.development of LC-MS/MS methods, data acquisition, and interpretation; S.M.C.acquisition and interpretation of toxicology data sets, histopathology, and TEM examinations; H.E.G.--design of experiments, data interpretation, supervision of experiments, co-wrote the paper, and funding acquisition; B.E.-conceived project, study design, design of synthesis and formulation experiments, supervision of experiments, data analysis and interpretation, co-wrote the paper and funding acquisition. All authors critically evaluated the paper prior to submission.

\section{Competing interests}

B.E. and H.E.G are cofounders of Exavir Therapeutics, Inc. and are inventors on patents that cover stable ProTides and the long-acting slow effective release formulations. The authors declare that this work was produced solely by the authors and that no other individuals or entities influenced any aspects of the work including, but not limited to the study conception and design, data acquisition, analyses, and interpretation, and writing of the paper. The authors further declare that they have received no financial compensation from any other third parties for any aspects of the published work. The remaining authors declare no competing interests.

\section{Additional information}

Supplementary information The online version contains supplementary material available at https://doi.org/10.1038/s41467-021-25690-5.

Correspondence and requests for materials should be addressed to Benson Edagwa.

Peer review information Nature Communications thanks Jessica Reynolds, Michaela Serpi, and the other anonymous reviewer(s) for their contribution to the peer review of this work. Peer reviewer reports are available.

Reprints and permission information is available at http://www.nature.com/reprints

Publisher's note Springer Nature remains neutral with regard to jurisdictional claims in published maps and institutional affiliations.

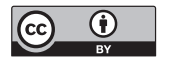

Open Access This article is licensed under a Creative Commons Attribution 4.0 International License, which permits use, sharing, adaptation, distribution and reproduction in any medium or format, as long as you give appropriate credit to the original author(s) and the source, provide a link to the Creative Commons license, and indicate if changes were made. The images or other third party material in this article are included in the article's Creative Commons license, unless indicated otherwise in a credit line to the material. If material is not included in the article's Creative Commons license and your intended use is not permitted by statutory regulation or exceeds the permitted use, you will need to obtain permission directly from the copyright holder. To view a copy of this license, visit http://creativecommons.org/ licenses/by/4.0/.

(c) The Author(s) 2021 